\title{
Optimal curing policy for epidemic spreading over a community network with heterogeneous population
}

\author{
STEFANIA OTTAVIANO \\ Fondazione Bruno Kessler, Via alla Cascata 56/d, 38123 Povo (Trento), Italy \\ Mathematics Department, University of Trento, Via Sommarive 14, 38123 Povo (Trento), Italy \\ FRANCESCO DE PELlEGRINI \\ Fondazione Bruno Kessler, Via alla Cascata 56/d, 38123 Povo (Trento), Italy \\ STEFANO BONACCORSI \\ Mathematics Department, University of Trento, Via Sommarive 14, 38123 Povo (Trento), Italy \\ AND \\ Piet VAN MiegheM \\ EEMCS, Delft University of Technology, Mekelweg 42628 CD Delft, Netherlands
}

[Received on 6 February 2018]

\begin{abstract}
The design of an efficient curing policy, able to stem an epidemic process at an affordable cost, has to account for the structure of the population contact network supporting the contagious process. Thus, we tackle the problem of allocating recovery resources among the population, at the lowest cost possible to prevent the epidemic from persisting indefinitely in the network. Specifically, we analyze a susceptible-infected-susceptible epidemic process spreading over a weighted graph, by means of a firstorder mean-field approximation. First, we describe the influence of the contact network on the dynamics of the epidemics among a heterogeneous population, that is possibly divided into communities. For the case of a community network, our investigation relies on the graph-theoretical notion of equitable partition; we show that the epidemic threshold, a key measure of the network robustness against epidemic spreading, can be determined using a lower-dimensional dynamical system. Exploiting the computation of the epidemic threshold, we determine a cost-optimal curing policy by solving a convex minimization problem, which possesses a reduced dimension in the case of a community network. Lastly, we consider a two-level optimal curing problem, for which an algorithm is designed with a polynomial time complexity in the network size.
\end{abstract}

Keywords: heterogeneous SIS model, community network, graph spectra, equitable partitions, convex optimization.

2000 Math Subject Classification: 82C20, 34A34, 34D23, 90C22, 90C25

\section{Introduction}

The diffusion and persistence of infectious diseases depend on complex interactions between individual units (namely people, cities, countries, etc.), the characteristics of a disease and, possibly, on the applied control policies. The last ones are aimed at arresting disease transmission or render the infection prevalence as low as possible.

Epidemic models have been used to describe a wide range of other phenomena as well, like social behaviors, diffusion of information, computer viruses, etc., indeed, although the basic mechanisms of 
these phenomena can be different, often their dynamical behavior can be described by the same type of equations [1]. One of the main objectives, in all these domains, is to gain insight into how the spreading process transmits and to identify the most effective strategies in order to prevent and control them.

In controlling the diffusion processes, the structure of the contact network plays a crucial role. In particular, several contact networks appear organized into communities. In this framework, a uniform control strategy not always represents the most effective way to reduce the infection rate, the number of affected individuals or the time of extinction $[2,3,4]$. Furthermore, curing costs may vary from node to node. In the case of community networks, curing costs may vary depending on the features of the specific community where curing controls are applied.

Thus, by taking into account the topology of a community network, in this work we want to determine a cost-optimal distribution of resources, that is able to prevent the disease from persisting indefinitely in the population. The non-uniform distribution of resources aims to control, in a cost-optimal way, the level of the nodes local curing rates. Increasing the curing rates of, e.g., some selected communities, is reflected into speeding up their detection capabilities and treatments (or, into installing better virus scan software, in the case of computer viruses) [2].

The problem of designing strategies to stop spreading processes in networks has been largely tackled. Though, in this context, to the best of our knowledge, very few works have described how to exploit the community structures in order to formulate an optimization problem for resources allocation, with lower complexity. Based on the theory of contact processes, Borgs et al. [4] characterize the optimal distribution of a fixed amount of antidote in a given network. Gourdin et al. [5] and Sahnen et al. [6] take advantage of the $N$-intertwined approximation [7] to analyze and control the spread of a SIS epidemic model. The same mean-field approach is adopted by Preciado et al in [8], where the authors propose a semidefinite programming (SDP) approach for optimal network immunization. Cost-optimal vaccine allocation in arbitrary undirected networks are obtained via the minimization of a vaccination cost function which depends on infection rates. In [9], the same authors specialize some specific instances of optimal network protection problem, via Geometric Programming techniques, to weighted, directed, strongly (and not necessarily strongly) connected networks to compute the cost and speed optimal allocation of vaccines and/or antidotes. In Sec. 3, we analyze more in detail the differences between their and our approach. Enyioha et al. [10] propose a distributed solution to the vaccine and antidote allocation problem to contain an epidemic outbreak in the absence of a central social planner. Each node locally computes its optimum investment in vaccine and antidotes needed to globally contain the spread of an outbreak, via local exchange of information with neighbors. Drakopoulos et. al [11,12] consider the propagation of an epidemic process over a network and study the problem of dynamically allocating a fixed curing budget to the nodes of the graph. The objective is to minimize the expected extinction time of the epidemics. In the case of bounded degree graphs, they provide a lower bound on the expected time to extinction under any such dynamic allocation policy.

\subsection{Outline and main results}

Compared to previous works on optimal curing policy, we are interested, particularly, in leveraging the subdivision of the population into communities. The motivation comes from the fact that community structures are a relevant non-trivial topological feature of complex networks. Community structures have been identified as a typical feature of social networks, tightly connected groups of nodes in the World Wide Web usually correspond to pages on common topics, whereas in the biology framework, e.g., in cellular and genetic networks, communities may relate to functional modules [13]. Consequently, 
in many practical situations, it appears reasonable to consider curing policies which apply per community (i.e., per hospital, school, village, or city, etc,...), rather than policies which apply per individual unit.

In particular, we consider a continuous-time susceptible-infected-susceptible (SIS) epidemic process, where an individual can be repeatedly infected, recover and yet be infected again. An input weighted graph captures the interaction between individuals and communities, where the heterogeneity, and possible asymmetries in the contagiousness, are caught by edge-dependent weights.

Our investigation, on a population divided into communities, has been based on the graph-theoretical notion of equitable partition [14, 15, 16]. A network with an equitable partition of its node set posses some interesting symmetry properties; we will use the word "symmetry" to refer to a certain structural regularity of the graph connectivity [16]. We take advantage of the notion of equitable partitions for providing curing policies, diversified for communities, capable to lead the system to extinction, at the minimum cost. In this context, our main goal is that we are able to formulate a convex cost minimization problem with a reduced dimension, with respect to the general case, where curing policies are providing for each node.

Spatial inhomogeneity has been incorporated in other models to study the epidemic control $[17,18$, 19], however not much effort has been made to explore inhomogeneous control strategies within this kind of models [2]. The problem of an inhomogeneous allocation of limited resources for a multi-group model, has been studied, instead, in [2] by Wan et al. The aim of the authors is to maximize the speed at which the virus is eliminated. Thus, considering a discrete-time epidemic model, they tackle the problem to minimize the dominant eigenvalue of a system matrix, subject to limiting constraints on some system parameters to be controlled. Compared to their formulation, we want to allocate resources to the communities, sufficient to lead the system towards the epidemic extinction, with the aim of minimizing a certain cost function. Moreover, in the cited paper, individuals transmit the disease through homogeneous mixing within their own group, as well as interactions with individuals in other groups, like in the usual metapopulation models. Such model is only a specific case of our network model, in fact, by the notion of equitable partition, we go beyond the full mesh assumption, within the communities, as well as outside, thus providing results for wider possible scenario (see Section 4).

The paper is organized as follows. In Sec. 2, first, we review some background concepts for epidemic processes on networks in the homogeneous setting and the related mean-field approximation adopted in the paper. Then, we provide the adaptation of the model to the heterogeneous setting and we report on the analysis of the global dynamics of the epidemic process. This allows to recognize the stability modulus of a matrix, encoding for the network structure and for the parameters of the model, as the critical value separating an absorbing phase, from an endemic phase. Leveraging on this result, in Sec. 3, we present the cost-optimal resource allocation problem. We use a semidefinite approach to formulate our optimization problem for the case of arbitrary undirected graphs with symmetric weights. Then, we show that this approach can be extended to some kind of not symmetric weighted networks, those whose adjacency matrix is diagonally symmetrizable. Moreover, for the case of a general directed weighted graph, we provide a suboptimal solution. In Sec 4 we consider the case when a contact network is shaped by an existing community network. First, we extend the results in [20] (related to equitable partitions in the case of undirected graphs), considering equitable partitions for directed weighted graphs. Specifically, we show how a certain kind of structure regularity, in a directed weighted graph, influences the system of differential equations that solve for the evolution in time of the approximated infection probability of each node. Then, we exploit such regularities in graph connectivity for reducing 
the original dynamical system to a lower dimensional one. By supposing that different curing rates can be chosen depending on the community network structure and that they can be optimized for a certain cost function, the latter system is used to reduce the dimension of our optimization problem. In the last part of the work, we propose a two-level optimal curing problem, that is, we have a two-dimensional curing policy, suitable, e.g., when the population can be divided in two categories (young and elders, male and female, etc,...). This kind of situation fits well certain networks with equitable partition, such as, e.g., bipartite graphs and interconnected star networks. In this case we provide a scalable bisection algorithm, that yields an $\varepsilon$-approximation of the optimal solution, in polynomial time in the input size. Finally, we carry out some numerical experiments. Proofs not included in previous sections for better readability are placed in Section 7.

\section{The epidemic network model}

In this section, we report some background concepts and new tools that we will use later to find a cost-optimal curing policy.

Let us consider a SIS epidemic process spreading over a simple undirected graph $G=(V, E)$, with edge set $E$ and node (vertex) set $V$. The order of $G$, denoted by $N$, is the cardinality of $V$. The edge set of $G$ consists of unordered pairs $\{i, j\}$, with $i, j \in V$, and $i \neq j$. Connectivity of graph $G$ is conveniently expressed by the symmetric $N \times N$ adjacency matrix $A$.

The viral state of a node $i$, at time $t$, is described by a Bernoulli random variable $X_{i}(t)$, where we set $X_{i}(t)=0$, if $i$ is healthy and $X_{i}(t)=1$, if $i$ is infected. Every node at time $t$ is either infected with probability $p_{i}(t)=\mathbb{P}\left(X_{i}(t)=1\right)$ or healthy (but susceptible) with probability $1-p_{i}(t)=\mathbb{P}\left(X_{i}(t)=0\right)$. In the homogeneous setting, the recovery process is a Poisson process with rate $\delta$, and the infection process is a per link Poisson process where the infection rate between an healthy and an infected node is $\beta$. All the infection and recovery processes are independent. The SIS process, developing a graph with $N$ nodes, can be modeled as a continuous-time Markov process with $2^{N}$ states, covering all possible combinations in which $N$ nodes can be infected [7]. The probability of the process of being in a certain state can be uniquely determined by the Kolmogorov's differential equations (i.e. a system of linear differential equations). However, the number of equations increases exponentially with the number of nodes; this poses several limitations in order to determines the set of solutions even for small network order. Hence, often, it is necessary to derive models that are an approximation of the exact original one $[7,21]$.

In this work, we consider a first order mean-field approximation (NIMFA), proposed by Van Mieghem et. al. in [7]. Basically, NIMFA replaces the original $2^{N}$ linear differential equations by $N$ non-linear differential equations representing the time-change of the infection probability of each node.

Epidemic threshold. For a network with finite order $N$, the exact SIS Markov process will always converge towards its unique absorbing state, that is the zero-state where all nodes are healthy. However, the process shows a phase transition behavior: indeed, there is a critical value $\tau_{c}$ of the effective spreading rate $\tau=\beta / \delta$, whereby if $\tau$ is lesser than $\tau_{c}$, the initial infection dies out quickly. Conversely, for $\tau$ larger than $\tau_{c}$, the infection spreading can last very long in any sufficiently large network [22, 23, 24]. The regime of persistent infection $\left(\tau>\tau_{c}\right.$ ), called the metastable or quasi-stationary state, is reached rapidly given an initial set of infected nodes and can persist for very long time [24]. In support of this, numerical simulations of SIS processes reveal that, even for fairly small networks $(N \simeq 100)$ and when $\tau>\tau_{c}$, the overall-healthy state is only reached after an unrealistically long time. Hence, the indication of the model is that, in the case of real networks, one should expect that above the epidemic threshold the extinction of epidemics is hardly ever attained [22, 23]. For this reason, the literature is mainly concerned with establishing the value of the epidemic threshold, being a key measure of the robustness 
against epidemic spreading.

In the homogeneous setting, NIMFA determines the epidemic threshold for the effective spreading rate as $\tau_{c}^{(1)}=\frac{1}{\lambda_{1}(A)}$, where $\lambda_{1}(A)$ is the spectral radius of the adjacency matrix $A$, (see $\left.[7,25]\right)$. When $\tau \leqslant \tau_{c}^{(1)}$ the only equilibrium of the NIMFA system is the zero point. When $\tau>\tau_{c}^{(1)}$, there exists a second nonzero steady-state that reflects well the observed viral behavior [26], and that can be regarded as the analogous of the quasi-stationary state of the exact stochastic SIS model. NIMFA yields an upper bound for the probability of infection of each node, as well as a lower bound for the epidemic threshold [7, 27]. This fact ensures that $\tau_{c}^{(1)}$ allows us to determine a safety region $\left\{\tau \leqslant \tau_{c}^{(1)}\right\}$ for the effective spreading rate, that guarantees the extinction of epidemics in a reasonable time frame. Thus, even though NIMFA is approximated, a design for our optimization problem, based on NIMFA, is always "safe" or "secure".

\subsection{Heterogeneous SIS mean-field model}

In this section, we consider a heterogeneous setting. We include the possibility that the infection rate is link specific, denoting by $\beta_{i j}$ the infection rate of node $j$ towards node $i$. Moreover, each node $i$ recovers at rate $\delta_{i}$, so that the curing rate is node specific. Basically, we allow for the epidemics to spread over a directed weighted graph.

A direct weighted graph (or weighted digraph) is a triple $G=(V, E, \rho)$, where the elements of $E$, named $\operatorname{arcs}$ (or directed edges), are ordered couples $e=(i, j)$ of distinct vertices of $V$, and $\rho: E \rightarrow(0, \infty)$ is a given function; $\rho(e)$ is called the weight of $e$. The matrix $A=\left(a_{i j}\right)$, with elements $a_{i j}=\rho(i, j)=\beta_{j i}$, is the weighted adjacency matrix of $G$. In our framework, $e=(i, j) \in E$ and $\rho(e)=\beta_{j i}$ means that node $i$ can infect node $j$ with rate $\beta_{j i}$. Again, self-loops and multiple edges (multiple arcs with the same direction) are not permitted. Hereafter, we shall assume that the directed graph is strongly connected, i.e., for all pairs of nodes $i, j \in V$, there is a path form $i$ to $j$ and from $j$ to $i$.

As in the homogeneous case, the SIS model with heterogeneous infection and recovery rates is a Markovian process. The time for infected node $j$ to infect any susceptible neighbor $i$ is an exponential random variable with mean $\beta_{i j}^{-1}$. Also, the time for node $j$ to recover is an exponential random variable with mean $\delta_{j}^{-1}$. A NIMFA model for the heterogeneous setting has been presented first in [28], where a node $i$ can infect all neighbors with the same infection rate $\beta_{i}$. Here we include the possibility that the infection rates depend on the connection between two nodes, thus covering a much more general case. The NIMFA governing equation for node $i$ in the heterogeneous setting writes as

$$
\frac{d p_{i}(t)}{d t}=\sum_{j=1}^{N} \beta_{i j} p_{j}(t)-\sum_{j=1}^{N} \beta_{i j} p_{i}(t) p_{j}(t)-\delta_{i} p_{i}(t), \quad i=1, \ldots, N .
$$

Let the vector $P(t)=\left(p_{1}(t), \ldots, p_{N}(t)\right)^{T}$ and let $\bar{A}=\left(\bar{a}_{i j}\right)$ be the matrix defined by $\bar{a}_{i j}=\beta_{i j}$ when $i \neq$ $j$, and $\bar{a}_{i i}=-\delta_{i}$; moreover let $F(P)$ be a column vector whose $i$-th component is $-\sum_{j=1}^{N} \beta_{i j} p_{i}(t) p_{j}(t)$. Then we can rewrite (2.1) in the following form:

$$
\frac{d P(t)}{d t}=\bar{A} P(t)+F(P)
$$

Let

$$
r(\bar{A})=\max _{1 \leqslant j \leqslant N} \operatorname{Re}\left(\lambda_{j}(\bar{A})\right),
$$


be the stability modulus [29] of $\bar{A}$, where $\operatorname{Re}\left(\lambda_{j}(\bar{A})\right)$ denotes the real part of the eigenvalues of $\bar{A}, j=$ $1, \ldots, N$. Now, we adopt a result from [29] that lead us to find the epidemic threshold, and to extend the global stability analysis of the homogeneous NIMFA system (see e.g. [20]) to the entirely heterogeneous setting, where each node can potentially infect each of its neighbors with different infection rates. We underline that to use the result in [29], the matrix $\bar{A}$ needs to be irreducible, this is equivalent to say that its associated digraph must be strongly connected.

THEOREM 2.1 If $r(\bar{A}) \leqslant 0$ then $P=0$ is a globally asymptotically stable equilibrium point in $I_{N}=[0,1]^{N}$ for the system (2.2), On the other hand if $r(\bar{A})>0$ then there exists a constant solution $P^{\infty} \in I_{N}-\{0\}$, such that $P^{\infty}$ is globally asymptotically stable in $I_{N}-\{0\}$ for (2.2).

Proof. See [29, Thm. 3.1].

REMARK 2.1 Let $A$ be an $N \times N$ irreducible and non negative matrix, $D$ a diagonal matrix with positive entries. Let $\sigma(A-D)$ be the spectrum of the matrix $A-D$, then the eigenvalue $\lambda \in \sigma(A-D)$, such that $\operatorname{Re}(\lambda)=r(A-D)$, is real (this follows also from (7.6)).

The result in Theorem 2.1 is crucial for the cost-optimal curing problem described in the next section. In fact, it identifies the value of the epidemic threshold, separating an absorbing phase, where the epidemics will go extinct, from an endemic phase. Thus, this critical threshold is recognized as a key value for treatment strategies against viral infection.

\section{Optimal curing policies for arbitrary weighted networks}

Now, leveraging on the result in Theorem 2.1, we address the problem of suppressing an epidemic spreading, by a cost-optimal distribution of resources within a networked population. Allocating more resources at each node aims to increase its curing rate, that is reflected, e.g., by speeding up its detection capabilities and treatments. We consider that recovery resources have an associated cost that might be different for each node. Thus, let us define a cost function which measures the expenditure in order to distribute curing resources to all nodes. Let $f_{i}\left(\delta_{i}\right)$ be a real, linear and monotonically increasing function with respect to $\delta_{i}$, whose value represents the effort of modifying the recovery rate of node $i$.

This model fits the case of disease treatment plans: policy makers can distribute different amount of resources (e.g. money for medicines, medical and nursery staff, etc,...) in a network of hospitals, or they can design a different health program for different districts, cities, or nations in the case of a timely mass prophylaxis plan. For instance, in the US, pharmaceutical supply caches and production arrangements have been pre-designated. This is done in order to be used for large-scale ongoing prophylaxis and/or vaccination campaigns in case of sudden intentional or natural outbreaks disease [30].

For now, we take into account an arbitrary weighted network. In Sec. 4, instead, we shall provide a cost-optimal curing policy for a network with community structure. Hereafter, we consider $f\left(\delta_{i}\right)=c_{i} \delta_{i}$, with $c_{i}>0$, that we shall call the cost coefficients, for $i=1, \ldots, N$. Thus, the cost function is the cumulative sum over the nodes' set

$$
U(\Delta)=\sum_{i=1}^{N} c_{i} \delta_{i}
$$

where $\Delta=\left(\delta_{1}, \ldots, \delta_{N}\right)$ is the curing rate vector. 


\subsection{Undirected graphs with symmetric weights.}

Now, let us assume that $\beta_{i j}=\beta_{j i}$, for all $i, j=1, \ldots, N$, i.e., the weighted adjacency matrix $A=\left(\beta_{i j}\right)$ is symmetric and, consequently, all its eigenvalues are real. Basically, now we are considering undirected graphs with symmetric weights.

Let us define the $N \times N$ curing rate matrix, $D=\operatorname{diag}(\Delta)$. We remark that, hereafter, we shall indicate with $\lambda_{1}(A)$ the maximum eigenvalue of $A$. By Theorem 2.1, we know that if $\lambda_{1}(A-\operatorname{diag}(\Delta)) \leqslant 0$, then the epidemics will go extinct. As we have explained in Section 2, the critical threshold for the mean-field model is a lower bound of the threshold of the exact Markov model. Thus, the condition $\lambda_{1}(A-\operatorname{diag}(\Delta)) \leqslant 0$ corresponds, in the exact stochastic model, to a region where the infectious process dies out exponentially fast for sufficiently large times [24]. We recall that, instead, above the exact threshold the overall-healthy state is reached only after an unrealistically long time. Hence, in order to find a cost-optimal distribution of resources that guarantees the extinction, we seek for the solution of the following problem.

Problem 3.1 (Eigenvalue Constraint Formulation) Find $\Delta \geqslant 0$ which solves

$$
\begin{array}{lc}
\text { minimize } & U(\Delta) \\
\text { subject to: } & \lambda_{1}(A-\operatorname{diag}(\Delta)) \leqslant 0, \quad \Delta \geqslant 0 .
\end{array}
$$

Problem 3.1 can be reformulated as a semidefinite program, that is a convex optimization problem [31]. In fact $\operatorname{diag}(\Delta)=\sum_{i=1}^{N} \Delta_{i} \operatorname{diag}\left(\mathbf{e}_{i}\right)$, where $\Delta_{i}$ is the $i$-th component of $\Delta$ and $\mathbf{e}_{i}$ is the $i$-th element of the standard basis so that $\operatorname{diag}\left(\mathbf{e}_{i}\right) \geqslant 0$. Hereafter, as in [32], the inequality sign in $M \geqslant 0$, when $M$ is a matrix, means that $M$ is positive semidefinite. Thus, we can express the optimization problem with eigenvalue constraint as a semidefinite programming (SDP) problem.

Problem 3.2 (Semidefinite Programming Formulation) Find $\Delta$ which solves

$$
\begin{array}{ll}
\text { minimize } & U(\Delta) \\
\text { subject to: } & \operatorname{diag}(\Delta)-A \geqslant 0 \\
& \Delta \geqslant 0
\end{array}
$$

The feasibility of the problem is always guaranteed, as showed in the following

Theorem 3.3 (Feasibility) Problem 3.2 is feasible.

Proof. We define $l_{\max }:=\max _{i} \sum_{j} a_{i j}$ and choose $\Delta=l_{\max } \mathbf{1}_{N}$, where $\mathbf{1}_{N}$ is the all-one vector of length $N$, consequently, $D=l_{\max } I_{N}$, with $I_{N}$ identity matrix of order $N$. Then, for any vector $w=\sum_{i=1}^{N} z_{i} v_{i}$, where $z_{i} \in \mathbb{R}$, for $i=1 \ldots N$ and $\left\{v_{1}, \ldots, v_{N}\right\}$ is an eigenvector basis of $A$, it holds

$$
w^{T}(A-D) w=w^{T}\left(\sum_{i=1}^{N} \lambda_{i}(A) z_{i} v_{i}-l_{\max } w\right) \leqslant\left(\lambda_{1}(A)-l_{\max }\right)\|w\|^{2} \leqslant 0,
$$

where the last inequality follows since $\lambda_{1}(A) \leqslant \max _{i} \sum_{j} a_{i j}$. Hence the chosen vector satisfies the constraint and we can assert that the feasible region is not empty.

Since the problem is feasible there is always an optimal point on the boundary [31] and, by the fundamental result of convex optimization, any locally optimal point of a convex problem is globally optimal [32, Sec. 4.4.2]. 
Existing results. As introduced in Sec. 1, an SDP approach is adopted also in [8] to detect a costoptimal distribution of protective resources in an arbitrary undirected network. Unlike our approach, they consider that each node $i$ can infect all its neighbors with the same infection rate $\beta_{i}$; moreover they describe the minimization of a decreasing vaccination cost function, which depends on the infection rates, that are allowed to be in a feasible interval. In the second part of the work they propose a greedy approach for the case of all-or-nothing vaccination, i.e., they restrict the infection rate to be in a discrete set, possibly different for each node, $\beta_{i} \in\left\{\underline{\beta_{i}}, \overline{\beta_{i}}\right\}$, where the two values, are fixed a priori.

With respect to their approach, in our model, each node can potentially infect each of its neighbors with different infection rates, thus we treat a wider scenario. In addition, from the next section onwards, we shall focus, mainly, on a population divided into communities, obtaining a dimensionality reduction of the optimization problem (3.2). Moreover, in Sec. 5.3 we propose a bisection algorithm for a two-level optimal curing problem, i.e we consider a two-dimension curing policy, providing that the population is divided in two categories, each of which will benefit from one of the two policies. The two available values of the curing rate are not fixed a priori.

At last, in [9], Preciado et al., leverage on Geometric Programming (GP) techniques for the resource allocation problem, applied to arbitrary weighted directed graphs, hence they do not require the symmetry of the adjacency matrix. However, the drawback of such formulation is that it does not fit for a linear cost function of the type we are considering, which is, anyway, a standard cost function of practical relevance. Thus, in the next section, we show how our formulation of the problem, involving a linear cost function, can be extended to a certain class of not symmetric matrices.

\subsection{Extension to directed weighted networks.}

The formulation of the optimization problem (3.2) holds for symmetric weighted adjacency matrix, however we shall show how it can be extended to a certain class of not symmetric matrices that are diagonally symmetrizable. In this case, for a not symmetric matrix $A$, there exists a diagonal matrix $G$ such that $G^{-1} A G$ is symmetric, for similarity their eigenvalues are the same and the semidefinite program formulation can be applied to $G^{-1} A G^{1}$. Thus, we can include also the case of not symmetric weighted adjacency matrix. A notable example is that of an undirected network where each node $i$ can infect all its neighbors with the same infection rate $\beta_{i}$ : the weighted adjacency matrix $B A$ with $A$ symmetric and $B=\operatorname{diag}\left(\beta_{i}\right)$ is not symmetric, however it is diagonally symmetrizable, indeed choosing $G=B^{1 / 2}$ we have $B^{-1 / 2}(B A) B^{1 / 2}=B^{1 / 2} A B^{1 / 2}$, which is symmetric (see, e.g., [8]). Hence, for our problem 3.2, we can request that the matrix $B^{1 / 2} A B^{1 / 2}$ is semidefinite positive.

Otherwise, if we have an arbitrary, strongly connected, directed weighted graph and a not symmetrizable $N$-dimensional adjacency matrix $A$, we can apply our formulation to its Hermitian part, $\mathscr{H}=$ $\left(A+A^{T}\right) / 2$, obtaining a suboptimal solution. More precisely, let $\operatorname{Re} \lambda(A)=\left(\operatorname{Re} \lambda_{1}(A), \cdots, \operatorname{Re} \lambda_{N}(A)\right)$ be the vector of the real part of the eigenvalues of $A$ and $\lambda(\mathscr{H}(A))=\left(\lambda_{1}(\mathscr{H}(A)), \cdots, \lambda_{N}(\mathscr{H}(A))\right)$, both arranged in decreasing order; then it holds $\operatorname{Re} \lambda(A) \prec \lambda(\mathscr{H}(A))$ [34, Thm. 10.28], meaning that $\lambda(\mathscr{H}(A))$ majorizes $\operatorname{Re} \lambda(A)$ [34, Sec. 10.1]. Basically, this result suggests that the stability modulus $\lambda_{1}(A-D)$ (see Remark 2.1) is smaller than $\lambda_{1}(\mathscr{H}(A)-D)$, hence the feasible region of our optimization problem, i.e., where $\lambda_{1}(\mathscr{H}(A)-D) \leqslant 0$, is a subset of the feasible region for the matrix $A$. Hence, if we solve the problem (3.2), choosing $\mathscr{H}(A)$, the value of the cost function obtained is an upper bound

\footnotetext{
${ }^{1}$ As suggested in [2], see [33] for a method to check if a matrix is symmetrizable, and, in case, the way to chose the diagonal matrix to achieve symmetry.
} 

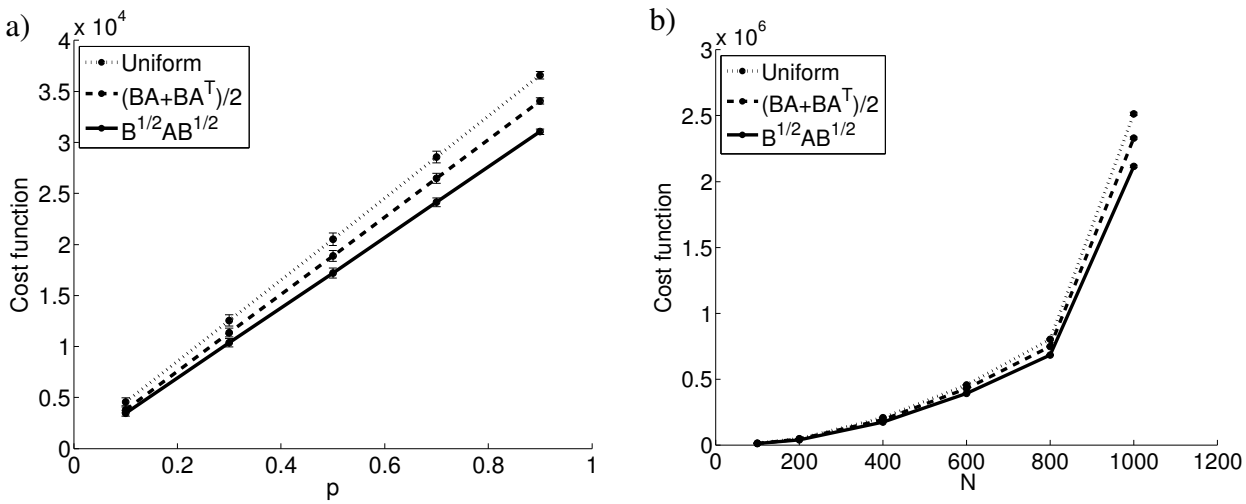

FIG. 1: Extension to directed weighted networks: comparison between optimal, suboptimal and uniform solutions. a) the cost values have been obtained averaging over 300 instances of Erdős-Rényi sample graphs with $N=100$, for different $p=0.1,0.3,0.5,0.7,0.9, \mathrm{~b}$ ) average over 300 Erdős-Rényi sample graphs with $N=100,200,400,600,800,1000$ for $p=0.3 ; 0.95$ confidence intervals are superimposed.

of the cost function that would be sufficient to bring $\lambda_{1}(A-D)$ at the critical value zero. Thus, we obtain a suboptimal solution, i.e., we will be able to lead the epidemic towards the extinction, but with more effort than it would be sufficient.

Hence, let us consider a diagonally symmetrizable weighted adjacency matrix $B A$; we want to compare the optimal cost function - corresponding to the optimal solution of the problem (3.2)) - with the suboptimal cost function, obtained considering the hermitian part of $B A$. Besides, we compute also the cost in the case of a uniform curing rate vector for which the maximum eigenvalue of $B^{1 / 2} A B^{1 / 2}$ attains zero. We use a standard solver for semidefinite programs (see [35]). In Fig. 1 a) we consider the cost functions obtained averaging over 300 instances of Erdős-Rényi random graphs with $N=100$ for increasing values of $p$. We take a matrix $B=\operatorname{diag}\left(\beta_{i}\right)$, where the infection rates are generated as uniform random variables in the interval $(0.1,3)$, and the $c_{i}$ 's constants in the interval $(0.5,5)$. We observe that the suboptimal cost function is close to the optimal cost function, the closer the lower the values of $p$. In Fig. 1 b), instead, we fix the value of $p=0.3$ and we plot the costs as functions of the number of nodes $N$. We can see a growth in the difference between the suboptimal and the optimal cost functions as the number of nodes increase. Ultimately, we obtain always an advantage in the use of suboptimal cost function with respect to the uniform case.

In the rest of the paper, we shall consider the case of a network with community structure. We shall show that - in order to find the epidemic threshold for the system (2.2) - it can be employed a matrix with lower dimension than the starting $N$-dimensional adjacency matrix. In turn, this provides a corresponding reduction in the dimension of our optimization problem (see Sec. 5).

\section{Community Networks}

Hereafter, we shall focus on the case when a contact network is shaped by an existing community network. This framework captures some of the most salient structural inhomogeneities in contact patterns in many applied contexts [36]. There exists an extensive literature on the effect of network community structures on epidemics. A specific community structure may arise due to, for example, geographic separation. Models utilizing this structure are commonly known as "metapopulation" models, where 

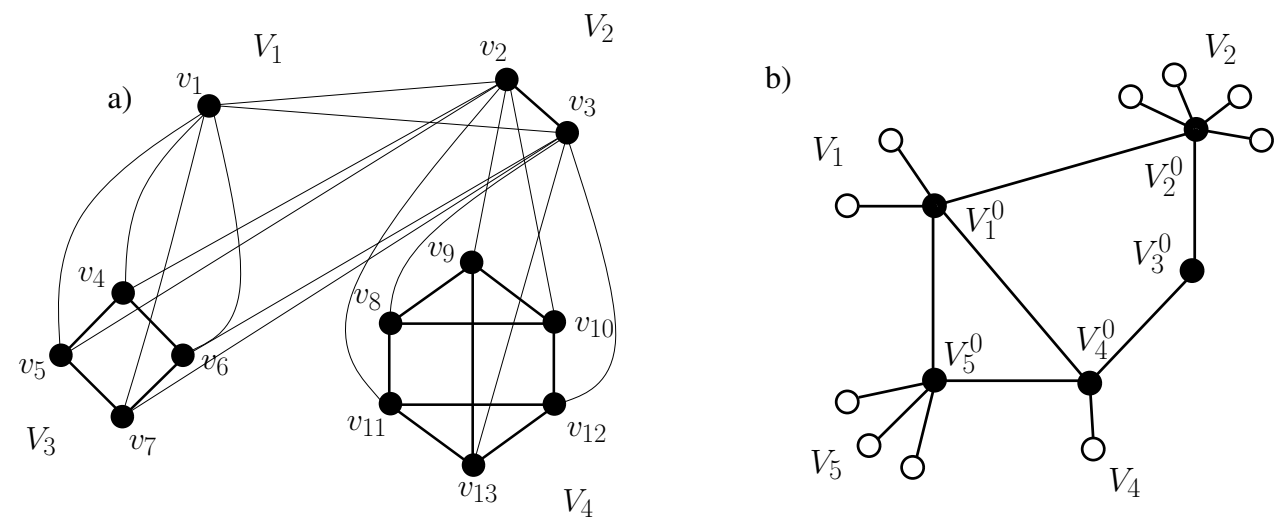

FIG. 2: A sample graphs with equitable partition. a) $V=\left\{V_{1}, V_{2}, V_{3}, V_{4}\right\}$, b) Interconnected star networks: $V=$ $\left\{V_{1}^{0}, V_{2}^{0}, V_{3}^{0}, V_{4}^{0}, V_{5}^{0}, V_{1}, V_{2}, V_{3}, V_{4}, V_{5}\right\}$

the population is compound of multiple interacting groups, which internally have homogeneous mixing [2] (see, e.g., [37, 38]). Such models assume that each community shares a common environment or is defined by a specific relationship. Some of the most common works on metapopulation regard a population divided into households with two level of mixing $([39,40,41])$. This model typically assumes that contacts, and consequently infections, between nodes in the same group occur at a higher rate than those between nodes in different groups [36]. Thus, groups can be defined, e.g., in terms of spatial proximity, considering that between-group contact rates (and consequently the infection rates) depend in some way on spatial distance, so that, each individual can be theoretically infected by each of the other individuals. However, models where infection can only be transmitted by nodes directly connected by an edge, may provide a more realistic approach to the study of the evolution of the epidemics. In turn, an important challenge is how to consider a realistic underlying structure and appropriately incorporate the influence of the network topology on the dynamics of epidemic [36, 42, 43, 44, 45].

In $[20,46]$ the authors analyze the dynamics of an epidemics on networks that are partitioned into local communities, through the first-order mean-field approximation discussed in Section 2. The investigation was based on the graph-theoretical notion of equitable partition $[14,15,16]$. Specifically, for an undirected graph, let $\pi=\left\{V_{1}, \ldots, V_{n}\right\}$ be a partition of the node set $V$, i.e., a sequence of mutually disjoint nonempty subsets of $V$, called cells, whose union is $V$, that we assume given a priori; $\pi$ is called equitable if the subgraph $G_{i}$ of $G(V, E)$ induced by $V_{i}$ is regular for all $i$ 's. Furthermore, for any two subgraphs $G_{i}$ and $G_{j}$, whenever there exists at least one connection between nodes in the first and second subgraph, then each node in $G_{i}$ is connected with the same number of nodes in $G_{j}$. In $[20,46]$ two-level infection rates have been considered: an intra-community infection rate and a lower inter-community infection rate. In the network structures hereafter, we generalize the model to more than two levels of infectiousness. We observe that usually a community is defined as a set of network nodes joined together in tightly-knit groups whereas among such group connections are looser [47]. Leveraging on the definition of equitable partition, instead, we can also consider that connections between nodes belonging to the same community can be, eventually, less dense than connections with other communities. Thus, the definition of community acquires a broader sense.

Networks with an equitable partition of the node set can describe models consisting of multiple 
smaller sub-populations such as, e.g., households, workplaces, or classes in a school, when the internal structure of each community is represented by a complete graph (members of a small community usually know each other) and all the nodes of adjacent communities are mutually linked (all member of adjacent communities may potentially come into contact). Equitable partitions can be observed also in the architecture of some computer networks where clusters of clients connect to single routers, whereas the router network has a connectivity structure with the nodal degree constrained by the number of ports (see as examples Fig. 2b). Equitable partitions appear also in the study of synchrony and pattern formation in coupled cell networks $[48,49]$ where they are named balanced partitions. Equitable partitions have been used also to analyze the controllability of multi-agent systems, for the case of a multi-leader setting [50], and for the leader-selection controllability problem, in characterizing the set of nodes from which a given networked control system (NCS) is controllable/uncontrollable [51]. These works show interesting realistic scenarios for the use of equitable partitions.

In particular, since the size of some real networks might pose limitations in our ability to investigate their spectral properties, we can leverage on the structural regularity of network with equitable partition, to reduce the dimensionality of our optimization problem (3.2).

Next, we define equitable partitions for the case of a directed weighted networks, extending the analysis in [20] to this framework. With a little abuse of notation, hereafter we shall refer to a partition of a network, to indicate the partition of its node set.

\subsection{Equitable partitions for weighted directed networks.}

The definition of equitable partitions can be extended to weighted directed graphs, based on [16, Def. 8.24]. That definition applies to oriented weighted graphs [16, Def. A.1]: we prefer to allow for a pair of symmetric oriented edges in order to cover naturally unoriented graphs.

Definition 4.1 Let $G=(V, E, \rho)$ be a weighted directed graph. The partition $\pi=\left\{V_{1}, \ldots, V_{n}\right\}$ of the node set $V$ is called inward equitable or outward equitable if for all $i, j \in\{1, \ldots, n\}$, there are

$$
c_{i j}^{i n} \in \mathbb{R} \quad \text { s.t. } \quad \sum_{w \in V_{j}} \rho((v, w))=c_{i j}^{i n}, \quad \text { for all } \quad v \in V_{i},
$$

or

$$
c_{i j}^{\text {out }} \in \mathbb{R} \quad \text { s.t. } \quad \sum_{w \in V_{j}} \rho((w, v))=c_{i j}^{\text {out }}, \quad \text { for all } \quad v \in V_{i}
$$

respectively. The partition is called equitable if it is both inward and outward equitable, hence for all $i, j \in\{1, \ldots, n\}$, there are

$$
c_{i j} \in \mathbb{R} \quad \text { s.t. } \quad \sum_{w \in V_{j}} \rho((v, w))+\rho((w, v))=c_{i j}, \quad \text { for all } \quad v \in V_{i} .
$$

We shall identify the set of all nodes in $V_{i}$ with the $i$-th community of the whole population.

REMARK 4.1 Let $k_{i}$ be the number of elements of $V_{i}, i=1, \ldots, n$. If the partition of the node set of a weighted di-graph is equitable, then for all $i, j \in\{1, \ldots, n\}$,

$$
k_{i} c_{i j}^{\text {out }}=k_{j} c_{j i}^{i n} \text {, }
$$

An equitable partition generates the quotient graph $G / \pi$, which is a multigraph, directed and weighted, with cells as vertices. For the sake of explanation, in the following, we will identify $G / \pi$ with the simple 
graph having the same vertex set, i.e. composed by the cells, where an edge exists between two cells, if at least one exists in the original multigraph.

For the purpose of modeling, nodes of the quotient graph can represent communities, e.g., villages, cities or countries. Link weights in the quotient graph, in turn, provide the strength of the contacts between such communities. In particular, the weight of a link may be (a non-negative) function of the number of people traveling per day between two countries; in fact, the frequency of contacts between them correlates with the propensity of a disease to spread between nodes.

Related to the quotient graph, there exists a quotient matrix, that contains the relevant structural information of the networks. Thus, let us consider the $n \times N$ matrix $S=\left(s_{i v}\right)$, where

$$
s_{i v}= \begin{cases}\frac{1}{\sqrt{\left|V_{i}\right|}} & v \in V_{i} \\ 0 & \text { otherwise }\end{cases}
$$

from which it follows that $S S^{T}=I_{n}$. Now let us consider the transpose of the adjacency matrix of the weighted directed graph $G$, that is

$$
A^{T}=\bar{A}+D,
$$

where, we remember, $\bar{A}$ is the matrix in (2.2) and $D=\operatorname{diag}(\Delta)$ is the curing rate matrix. Then, the transpose of the quotient matrix of $G$ (with respect to the given partition) is

$$
Q^{T}:=S A^{T} S^{T} .
$$

We can write the following explicit expression for $Q^{T}$ :

$$
Q^{T}=\operatorname{diag}\left(c_{i i}^{\text {out }}\right)+\left(\frac{k_{i} c_{i j}^{\text {out }}}{\sqrt{k_{i} k_{j}}}\right)_{i, j=1, \ldots, n}
$$

By (4.1), we can write the matrix $Q^{T}$ as

$$
Q^{T}=\operatorname{diag}\left(c_{i i}^{\text {out }}\right)+\left(\sqrt{c_{i j}^{\text {out }} c_{j i}^{\text {in }}}\right)_{i, j=1, \ldots, n}
$$

REMARK 4.2 We observe that matrix $Q$ in (4.4) might not be symmetric, whereas in the case of undirected graphs it is always symmetric (see, e.g., $[15,20]$ ). Even though we have represented the most general definition of an equitable partition simpler situations can be represented. E.g., nodes of the same community may infect all nodes in another community with the same rate.

When considering a population partitioned into communities, it may be appropriate to take into account the case where all nodes of a tagged community $j$ have the same recovery rate $\delta_{j}, j=1, \ldots, n$. In turn, such rate may differ from one community to the other. We remember that $N$ is the total number of nodes in the network, whereas $n$ is the number of communities.

DEFINITION 4.2 Let us introduce the $1 \times n$ vector of nonzero curing rates $\bar{\Delta}=\left(\overline{\boldsymbol{\delta}}_{1}, \ldots, \overline{\boldsymbol{\delta}}_{n}\right)$, that we shall call the reduced curing rate vector and $\bar{D}=\operatorname{diag}(\bar{\Delta})$, the reduced curing rate matrix.

Thus, we have the $1 \times N$ curing rates vector $\Delta=\left(\delta_{1}, \ldots, \delta_{N}\right)$, with components $\delta_{z}=\bar{\delta}_{j}$ for all $z \in V_{j}$ and $j=1, \ldots, n$. 
In appendix 7.1 we shall discuss when and how it is possible to reduce the original system (2.2) to a system of $n$ differential equations, through the matrix $Q^{T}$. Since for our optimization problem the parameter of interest is the epidemic threshold, in this section we limit our self to results related to this critical value.

LEMma 4.1 Let $\pi=\left\{V_{1}, \ldots, V_{n}\right\}$ be an equitable partition. Let $A^{T}$ and $Q^{T}$ be weighted matrices as in (4.2) and (4.4), respectively. Then, it holds:

i) $\left(A^{T}-D\right) S^{T}=S^{T}\left(Q^{T}-\bar{D}\right)$.

ii) For all $\lambda \in \mathbb{C}$ and all $x \in \mathbb{C}^{n}$

$$
\left(Q^{T}-\bar{D}\right) x=\lambda x \quad \text { if and only if } \quad\left(A^{T}-D\right) S^{T} x=\lambda S^{T} x .
$$

Now let us consider the system of $N$ differential equations (2.2). It is possible to extend [20, Thm. 4.1] to the case of directed graphs. Following that result, if we assume that at time $t=0$ the infection probability is equal for all nodes in the same community (while it may differ from one community to the other), the number of equations in (2.2) can be reduced by using the transpose of the quotient matrix $Q^{T}$. Hence, the reduced dynamical system writes

$$
\frac{d \bar{p}_{j}(t)}{d t}=\left(1-\bar{p}_{j}(t)\right) \sum_{\substack{m=1 \\ m \neq j}}^{n} c_{j m}^{\text {out }} \bar{p}_{m}(t)+c_{j j}^{\text {out }}\left(1-\bar{p}_{j}(t)\right) \bar{p}_{j}(t)-\bar{\delta}_{j} \bar{p}_{j}(t), \quad j=1, \ldots, n
$$

where $\bar{p}_{j}(t)$ is the infection probability of a node in the community $j$. We can prove that, in the case of a graph whose node set has an equitable partition, and regardless of initial conditions, the critical threshold for (2.2), applying Thm. 2.1, can be determined directly considering the reduced system (7.1).

Proposition 4.3 The elements of the curing rates vector $\Delta=\left(\delta_{1}, \ldots, \delta_{N}\right)$, that determine the critical threshold of (2.2), are identified by the elements of $\bar{\Delta}=\left(\bar{\delta}_{1}, \ldots, \bar{\delta}_{n}\right)$, in such a way that $\delta_{z}=\bar{\delta}_{j}$ for all $z \in V_{j}, j=1, \ldots, n$, for which

$$
r\left(Q^{T}-\bar{D}\right)=0
$$

where $r$ is the stability modulus.

Since the quotient matrix and the adjacency matrix have the same stability modulus (and so their transposed do), a computational advantage can be obtained in the calculation of the critical threshold of the system (2.2). This result is very relevant for our optimization problem. Indeed, in the case of a network with equitable partition, we can use a lower dimensional matrix to compute the epidemic threshold.

\section{Optimization for Networks with Equitable Partitions}

In this section, we consider a heterogeneous curing control per community. First, we assume that all nodes in community $j$ infect all nodes in community $i$ with the same infection rate, $\beta_{V_{i} V_{j}}$, and that $\beta_{V_{i} V_{j}}=\beta_{V_{j} V_{i}}, i, j=1, \ldots, n$. In this case, the graph is undirected and the weights are symmetric, thus the quotient matrix $Q$ is symmetric and has real eigenvalues. Now let us consider the $1 \times n$ reduced curing rate vector $\bar{\Delta}$, the cost function writes

$$
U(\bar{\Delta})=\sum_{j=1}^{n} c_{j} k_{j} \bar{\delta}_{j}
$$


Thus, $U(\bar{\Delta})$ is the cost for curing all elements of each community $j$ at rate $\bar{\delta}_{j}$, where $c_{j}>0, j=$ $1, \ldots, n$. We seek for the solution of the following

Problem 5.1 (Eigenvalue Constraint Formulation) Find $\bar{\Delta} \geqslant 0$ which solves

$$
\begin{array}{lc}
\text { minimize } & U(\bar{\Delta}) \\
\text { subject to: } & \lambda_{1}(Q-\operatorname{diag}(\bar{\Delta})) \leqslant 0, \quad \bar{\Delta} \geqslant 0
\end{array}
$$

which also writes

Problem 5.2 (Semidefinite Programming Formulation) Find $\bar{\Delta} \geqslant 0$ which solves

$$
\begin{array}{lc}
\text { minimize } & U(\bar{\Delta}) \\
\text { subject to: } & \operatorname{diag}(\bar{\Delta})-Q \geqslant 0 \\
& \bar{\Delta} \geqslant 0
\end{array}
$$

Thm. 3.3 guarantees the feasibility of the problem. The general case of equitable partitions introduced in Sec. 4.1, may not lead to a symmetric quotient matrix $Q$. However, we may consider suboptimal solutions - as explained in Sec. 3.1 - obtained by applying the formulation of our optimization problem to the hermitian part of $Q$.

In the next section, we consider a simpler version of Problem 5.2 and we design a more efficient algorithm with respect to the SDP program.

\subsection{Two-level curing problem}

The state of the art for SDP solvers such as, e.g., the SDP T3 solver used for our numerical computation, provide solutions for moderate size graphs. Actually, the best known bound for the complexity of an $\varepsilon$ solution attained with an interior point method is $O\left(n^{3.5} \log (1 / \varepsilon)\right)$, where $\varepsilon$ represents the accuracy [52]. The problem can be solved more efficiently when we face a two-level optimal curing problem, for which we shall provide an algorithm that yields an $\varepsilon$-approximation of the optimal solution, with a complexity equal to $O\left(\log (n) n^{3.3731} \log (1 / \varepsilon)\right)$ (see Thm. 5.7). Precisely, we consider only two possible levels of the nodes local curing rates, let us say $\delta_{0}$ and $\delta_{1}$, that are not fixed a priori. This situation fits well, e.g., in the case of a network where communities are of "two types". Communities of the first type are eligible for curing rate $\delta_{0}$, whereas communities of the second type are eligible for curing rate $\delta_{1}$. For convenience, we define the former, central communities, and the latter, terminal communities.

Such kind of configuration is suitable for a network that is, e.g., bipartite (where each node, e.g., represents a full-meshed community), or for an interconnected stars network, i.e., a network obtained by interconnecting star graphs by linking stars' central nodes (see Fig 2b). Let us note that the BarabásiAlbert graph model [53], that captures the power-law degree distribution often seen (or approximately seen) in real-world networks, can be regarded as a set of hubs with star graph features [54]. Bipartite networks, instead, can be used to understand the spreading of sexually transmitted diseases, in which the population is naturally divided into males and females and the disease can only be transmitted between nodes of different kinds. Bipartite networks can also represent the spreading of diseases in hospitals, in which one type of node accounts for (isolated) patients and the other type for caregivers, or some vectorborne diseases, such as malaria, in which the transmission can only take place between the vectors and the hosts [1]. 
Thus, let us consider the following partition of the node set, $\pi_{0}=\left\{V_{1}^{0}, \ldots, V_{m}^{0}\right\}$ and $\pi_{1}=\left\{V_{1}, \ldots, V_{m^{\prime}}\right\}$. We assume that the node set partition $\pi=\pi_{0} \cup \pi_{1}$ is equitable. Let us introduce the curing matrix $D=\operatorname{diag}\left(\delta_{0} \mathbf{1}_{m}, \boldsymbol{\delta}_{1} \mathbf{1}_{m^{\prime}}\right)$ and define

$$
I_{m}^{0}=\left[\begin{array}{cc}
I_{m} & 0 \\
0 & 0
\end{array}\right], \quad I_{m^{\prime}}^{1}=\left[\begin{array}{cc}
0 & 0 \\
0 & I_{m^{\prime}}
\end{array}\right]
$$

where $I_{m}$ is the identity matrix of order $m$. Then, we can write the semidefinite programming for the two-level curing rates, shortly the $2 D$ curing problem, as follows:

Problem 5.3 (Semidefinite Programming 2D Formulation) Find $\Delta_{2}=\left(\delta_{0}, \delta_{1}\right)$ which solves

$$
\begin{array}{ll}
\operatorname{minimize} & \multicolumn{1}{c}{U\left(\Delta_{2}\right)} \\
\text { subject to: } & \delta_{0} I_{m}^{0}+\delta_{1} I_{m^{\prime}}^{1}-Q \geqslant 0 \\
& \Delta_{2} \geqslant 0
\end{array}
$$

The cost function is

$$
U\left(\Delta_{2}\right)=\sum_{V_{j} \in \pi_{0}} k_{j} f_{0}\left(\delta_{0}\right)+\sum_{V_{z} \in \pi_{1}} k_{z} f_{1}\left(\delta_{1}\right),
$$

where $f\left(\delta_{0}\right)=c_{0} \delta_{0}$, and $f_{1}\left(\delta_{1}\right)=c_{1} \delta_{1}$, with $c_{0}, c_{1}>0$, represent the effort to modify the recovery rate for nodes belonging to $V_{j} \in \pi_{0}$, and $V_{z} \in \pi_{1}$, respectively.

In Section 7.5 of the Appendix, we shall provide some simple examples for the optimal solution of the Problem (5.3).

\subsection{Properties of the $2 D$ curing problem}

In the design of our algorithmic solution, we have leveraged on some basic properties of the $2 D$ curing problem. In order to do so, we need a few basic facts recalled next.

Proposition 5.4 Let $A$ be an $n \times n$ symmetric, irreducible and non negative matrix and let $D=$ $\operatorname{diag}\left(\delta_{1}, \ldots, \delta_{n}\right)$ :

i. if $\delta_{i}=0$ for some $i=1, \ldots n$, then $\lambda_{1}(A-D) \geqslant 0$

ii. The function $\left(\delta_{1}, \ldots, \delta_{n}\right) \longmapsto \lambda_{1}(A-D)$ is continuous;

iii. $\lambda_{1}(A-D)$ is strictly decreasing in $\delta_{i}, i=1, \ldots, n$.

Let us denote by $\Gamma=\left\{\left(\delta_{0}, \delta_{1}\right) \mid \lambda_{1}\left(\operatorname{diag}\left(\delta_{0} \mathbf{1}_{m}, \delta_{1} \mathbf{1}_{m^{\prime}}\right)-Q\right) \geqslant 0\right\}$ the feasibility region of Prob. 5.3: same argument of Thm. 3.3 let us state that it is non empty; furthermore, the problem structure guarantees $\Gamma$ to be convex [32]. We indicate $\Gamma_{0}$ and $\Gamma_{1}$ the standard projections of $\Gamma$ onto the $\delta_{0}$-axis and the $\delta_{1}$-axis, respectively.

LEMma 5.1 (Monotonicity) Let $\phi: \delta_{0} \longmapsto \phi\left(\delta_{0}\right)$ be the function that associates to $\delta_{0} \in \Gamma_{0}$ the value $\delta_{1}=\phi\left(\delta_{0}\right) \in \Gamma_{1}$ such that $\lambda_{1}\left(Q-\operatorname{diag}\left(\delta_{0} \mathbf{1}_{m}, \delta_{1} \mathbf{1}_{m^{\prime}}\right)\right)=0$. Then, $\phi$ is decreasing.

Proof. First, let us show that $\phi$ is a well defined function over $\Gamma_{0}$. Let $\delta_{0} \in \Gamma_{0}$, because of feasibility, there exists $\overline{\boldsymbol{\delta}}_{1}$, where $\left(\delta_{0}, \overline{\boldsymbol{\delta}}_{1}\right) \in \Gamma$, such that $\lambda_{1}\left(Q-\operatorname{diag}\left(\delta_{0} \mathbf{1}_{m}, \overline{\boldsymbol{\delta}}_{1} \mathbf{1}_{m^{\prime}}\right)\right) \leqslant 0$. Furthermore, it holds $\lambda_{1}\left(Q-\operatorname{diag}\left(\delta_{0} \mathbf{1}_{m}, 0 \mathbf{1}_{m^{\prime}}\right)\right) \geqslant 0$ by $\left.i\right)$ of Prop. 5.4. Because of ii) and iii) in Prop. 5.4 we know that $\lambda_{1}\left(Q-\operatorname{diag}\left(\delta_{0} \mathbf{1}_{m}, \delta_{1} \mathbf{1}_{m^{\prime}}\right)\right)$ is a continuous strictly decreasing function of $\delta_{1}$ over $\left[0, \overline{\boldsymbol{\delta}}_{1}\right]$, so there exists one and only one value $\delta_{1} \in \Gamma_{1}$ satisfying the definition of $\phi$. 
Let $z>0$ and assume that $\phi\left(\delta_{0}+z\right)=\phi\left(\delta_{0}\right)+\zeta>\phi\left(\delta_{0}\right)$, for some $\zeta>0$, i.e, that $\phi$ is not decreasing. From the definition of $\phi$ there exists $0 \neq w \in \operatorname{ker}\left(\operatorname{diag}\left(\left(\left(\delta_{0}+z\right) \mathbf{1}_{m}, \phi\left(\delta_{0}+z\right) \mathbf{1}_{m^{\prime}}\right)-Q\right)\right.$. Hence, we can write

$$
\begin{aligned}
& w^{T}\left(Q-\operatorname{diag}\left(\boldsymbol{\delta}_{0} \mathbf{1}_{m}, \phi\left(\boldsymbol{\delta}_{0}\right) \mathbf{1}_{m^{\prime}}\right)\right) w=w^{T} \operatorname{diag}\left(z \mathbf{1}_{m}, \zeta \mathbf{1}_{m^{\prime}}\right) w+w^{T}\left(Q-\operatorname{diag}\left(\left(\boldsymbol{\delta}_{0}+z\right) \mathbf{1}_{m}, \phi\left(\boldsymbol{\delta}_{0}+z\right) \mathbf{1}_{m^{\prime}}\right)\right) w \\
& =w^{T} \operatorname{diag}\left(\left(z \mathbf{1}_{m}, \zeta \mathbf{1}_{m^{\prime}}\right)\right) w>0,
\end{aligned}
$$

where the strict inequality holds because $\operatorname{diag}\left(z \mathbf{1}_{m}, \zeta \mathbf{1}_{m^{\prime}}\right)>0$. Since $\lambda_{1}\left(Q-\operatorname{diag}\left(\delta_{0} \mathbf{1}_{m}, \phi\left(\delta_{0}\right) \mathbf{1}_{m^{\prime}}\right)=0\right.$, this means that $Q-\operatorname{diag}\left(\delta_{0} \mathbf{1}_{m}, \phi\left(\delta_{0}\right) \mathbf{1}_{m^{\prime}}\right)$ must be semidefinite negative and we have a contradiction.

We prove next that the search for the optimal solution can be restricted to a compact subset of $\Gamma$.

TheOREM 5.5 (Compact search set) There exist two pairs $\left(\delta_{0}^{\min }, \delta_{0}^{\max }\right)$ and $\left(\delta_{1}^{\min }, \delta_{1}^{\max }\right)$ such that a solution $\Delta_{2}^{*}=\left(\delta_{0}^{*}, \delta_{1}^{*}\right)$ of Prob. 5.3 belongs to a compact subset $\Gamma^{\prime} \subseteq\left[\delta_{0}^{\min }, \delta_{0}^{\max }\right] \times\left[\delta_{1}^{\min }, \delta_{1}^{\max }\right]$.

Proof. Let us define $\hat{c}_{0}=\sum_{V_{j} \in \pi_{0}} c_{0} k_{j}$ and $\hat{c}_{1}=\sum_{V_{z} \in \pi_{1}} c_{1} k_{z}$, then we write $U_{l_{\max }}=\hat{c}_{0} l_{\max }+\hat{c}_{1} l_{\max }$, with $l_{\text {max }}$ as in Thm. 3.3, and $U^{*}=\hat{c}_{0} \delta_{0}^{*}+\hat{c}_{1} \delta_{1}^{*}$. Let us denote $\Delta_{2}^{l_{\max }}=\left(l_{\max }, l_{\max }\right)$, by Thm. 3.3, $\Delta_{2}^{l_{\max }} \in \Gamma$, hence $U_{l_{\max }} \geqslant U^{*}$ and, by defining set $\left.\Omega=\left\{\left(\delta_{0}, \delta_{1}\right): \hat{c}_{0} \delta_{0}+\hat{c_{1}} \delta_{1} \leqslant U_{l_{\max }}\right)\right\}$, it follows that $\left(\delta_{0}^{*}, \delta_{1}^{*}\right) \in \Gamma^{\prime}=\Gamma \cap \Omega ; \Gamma^{\prime}$ is closed as intersection of closed sets.

Now, feasibility conditions of Prob. 5.3 require matrix $Q-\left(\delta_{0} I_{m}^{0}+\delta_{1} I_{m^{\prime}}^{1}\right)$ to be semidefinite negative. We define $f\left(\delta_{0}\right)=\lambda_{1}\left(Q-\left(\delta_{0} I_{m}^{0}+\left(\frac{U_{l_{\max }-\hat{c}_{0} \delta_{0}}}{\hat{c}_{1}}\right) I_{m^{\prime}}^{1}\right)\right)$ : we have $f\left(l_{\max }\right) \leqslant 0$ since $\left(l_{\max }, l_{\max }\right) \in \Gamma$ and $f(0) \geqslant 0$ by $i$ ) of Prop. 5.4. By assertion ii) in Prop. 5.4, $f\left(\delta_{0}\right)$ is a continuous function. Hence, there exists $\delta_{0}^{\min }$ such that $f\left(\delta_{0}^{\min }\right)=0$, and since $\phi$ is decreasing $\phi\left(\delta_{0}^{\min }\right)=\delta_{1}^{\max }$. We can repeat the same reasoning by inverting the role of $\delta_{1}$ and $\delta_{0}$ defining $g\left(\delta_{1}\right)=\lambda_{1}\left(Q-\left(\left(\frac{U_{\max }-\hat{c}_{1} \delta_{1}}{\hat{c}_{0}}\right) I_{m}^{0}+\delta_{1} I_{m^{\prime}}^{1}\right)\right)$. Hence, we can assert that exists $\delta_{1}^{\min }$ such that $g\left(\delta_{1}^{\min }\right)=0$ and $\phi\left(\delta_{1}^{\min }\right)=\delta_{0}^{\max }$.

Finally, by letting $r: \hat{c}_{0} \delta_{0}+\hat{c}_{1} \delta_{1}=U_{l_{\max }}$, the points $\left(\delta_{0}^{\min }, \delta_{1}^{\max }\right)$ and $\left(\delta_{0}^{\max }, \delta_{1}^{\min }\right)$ belong to $\partial \Gamma \cap r$, i.e., they belong to $\partial \Gamma^{\prime}$, so $\Gamma^{\prime} \subseteq\left[\delta_{0}^{\min }, \delta_{0}^{\max }\right] \times\left[\delta_{1}^{\min }, \delta_{1}^{\max }\right]$, and consequently, being $\Gamma^{\prime}$ closed, it is also compact.

REMARK 5.1 Thm. 5.5 allows us to identify an interval of the values of $\delta_{0}$ and $\delta_{1}$ where we can restrict the search of $\left(\delta_{0}^{*}, \delta_{1}^{*}\right)$. Since $\Gamma^{\prime} \subseteq\left[\delta_{0}^{\min }, \delta_{0}^{\max }\right] \times\left[\delta_{1}^{\min }, \delta_{1}^{\max }\right]$ and $\left(\delta_{0}^{*}, \delta_{1}^{*}\right) \in \Gamma^{\prime}$, then $\delta_{0}^{*} \in\left[\delta_{0}^{\min }\right.$, $\left.\delta_{0}^{\max }\right]$ and $\delta_{1}^{*} \in\left[\delta_{1}^{\min }, \delta_{1}^{\max }\right]$. This is one key property in the algorithmic search of the optimal solution presented in the following section.

Finally, a direct proof that the optimal solution lies on $\partial \Gamma^{\prime}$ follows:

Corollary 5.1 A solution $\Delta_{2}^{*}=\left(\delta_{0}^{*}, \delta_{1}^{*}\right)$ of Prob. 5.3 belongs to $\partial \Gamma^{\prime} \cap \Omega$.

Proof. Let us assume $\Delta_{2}^{*}=\left(\delta_{0}^{*}, \delta_{1}^{*}\right) \in \Gamma^{\prime} \backslash \partial \Gamma^{\prime}$. $\Delta_{2}^{*}$ is feasible, hence $\lambda_{1}(Q-D)<0$, with $D=$ $\operatorname{diag}\left(\delta_{0}^{*} \mathbf{1}_{m}, \delta_{1}^{*} \mathbf{1}_{m^{\prime}}\right)$. From Prop. 5.4, again we can find $0<\delta_{1}^{\prime}<\delta_{1}^{*}$ such that $\lambda_{1}\left(Q-\operatorname{diag}\left(\delta_{0}^{*} \mathbf{1}_{m}, \delta_{1}^{\prime} \mathbf{1}_{m^{\prime}}\right)\right)=$ 0 , where, i.e., $\Delta_{2}^{\prime}=\left(\delta_{0}^{*}, \delta_{1}^{\prime}\right) \in \partial \Gamma^{\prime}$. But, $U\left(\Delta_{2}^{*}\right)-U\left(\Delta_{2}^{\prime}\right)=\hat{c}_{1}\left(\delta_{1}^{*}-\delta_{1}^{\prime}\right)>0$. Contradiction.

\subsection{Bisection Algorithm}

Tab. 1 reports on the pseudocode of algorithm OptimalThreshold2D: it solves the $2 D$ curing problem. It employs three additional functions LeftCorner (Tab. 2) RightCorner and, finally, BisectionThreshold (Tab. 3). 
Table 1: Optimal Threshold2D: solves the $2 D$ optimal curing problem via the bisection search.

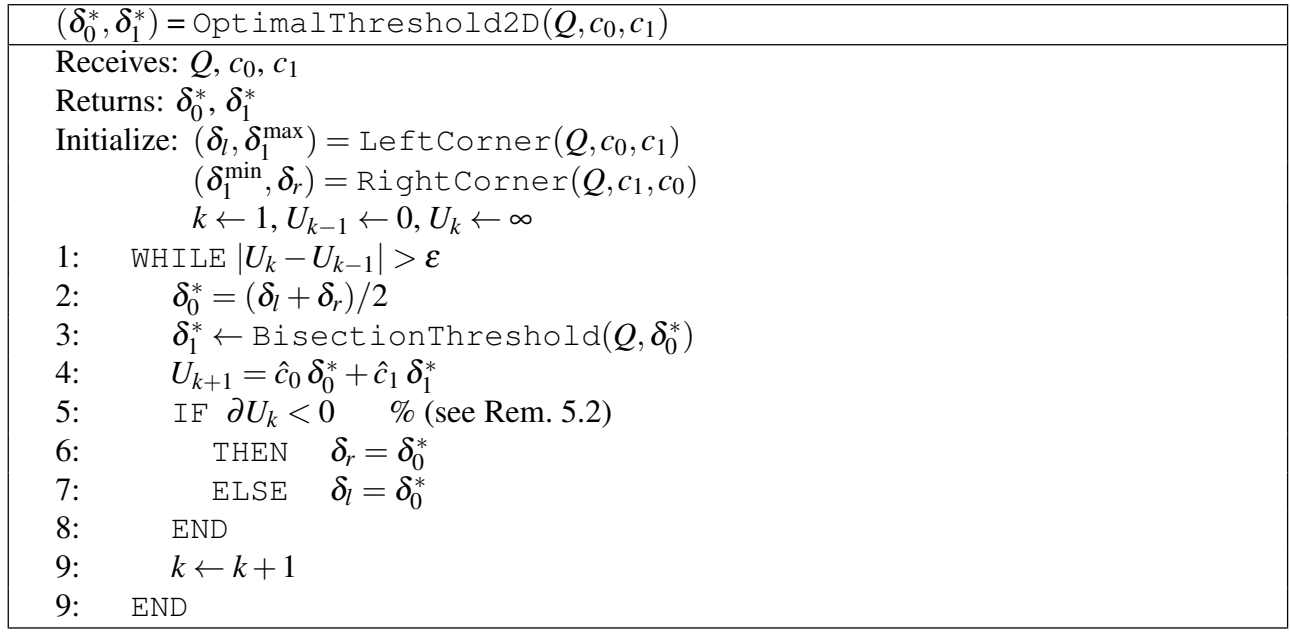

LeftCorner identifies via bisection feasible point $\left(\delta_{0}^{\min }, \delta_{1}^{\max }\right)$; the bisection search operated by LeftCorner - see proof of Thm. 5.5 - is performed along values $\delta_{1}=f\left(\delta_{0}\right)$. The companion function RightCorner identifies the point $\left(\delta_{0}^{\max }, \delta_{1}^{\min }\right)$; the pseudocode is omitted for the sake of space.

Procedure isNegativeDefinite is the standard test for a real symmetric matrix $A$ to be negative definite; it requires to verify $\operatorname{sgn}\left(\operatorname{det}\left(A_{k}\right)\right)=(-1)^{k}$ where $A_{k}$ is the $k$-th principal minor of $A$, i.e., the matrix obtained considering the first $k$ rows and columns only. Finally, the OptimalThreshold2D algorithm performs a bisection search based on a subgradient descent over the utility function $U\left(\delta_{0}\right)=$ $\hat{c}_{0} \delta_{0}+\hat{c}_{1} \phi\left(\delta_{0}\right)$.

REMARK 5.2 In Tab. 1 we have reported an implementation assuming the calculation of the subgradient $\partial U$ at each mid point $x$. However, it is sufficient to evaluate the increment at a point $x+\varepsilon_{1}$ within the feasibility region for some $\varepsilon_{1}>0$ : if $U(x)<U\left(x+\varepsilon_{1}\right)$, then, due to convexity, the whole interval $\left[x+\varepsilon_{1},+\infty\right)$ can be discarded. Conversely, if $U(x)>U\left(x+\varepsilon_{1}\right)$, then, due to convexity, the whole interval $[0, x)$ can be discarded during the search. This operation can be performed at a cost $O(1)$ when $U(x)$ and $U\left(x+\varepsilon_{1}\right)$ are known, i.e., at the cost of two calls of BisectionThreshold.

We note that REPEAT loop stops when $\varepsilon>\prod\left|\lambda_{i}\right|=|\operatorname{det}(Q-D)|>\left|\lambda_{1}\right|^{n}$, i.e., when $\left|\lambda_{1}\right|<(\varepsilon)^{1 / n}$. Furthermore, the termination condition in BisectionThreshold, LeftCorner and Right Corner requires $\Delta_{2}$ to lie within the feasible region and the determinant to be smaller than $\varepsilon$.

THEOREM 5.6 (Correctness) OptimalThreshold2D is an $\varepsilon$-approximation of an optimal solution.

Proof. The algorithm operates a bisection search for a global minimum of $U\left(\Delta_{2}\right)=\hat{c}_{1} \delta_{0}+\hat{c}_{1} \phi\left(\delta_{0}\right)$, where $U\left(\Delta_{2}\right)$ is a convex function. Let $V=U_{l_{\max }}$ and $\Delta_{2}^{*}$ be the optimal solution: from the properties of the bisection search on (quasi-)convex functions [32][Ch. 4, pp. 145], the accuracy at step $r=$ $\left\lceil\log _{2}(V / \varepsilon)\right\rceil$ of the algorithm is $\left|U_{r}-U\left(\Delta_{2}^{*}\right)\right|<V 2^{-r}<\varepsilon$.

Furthermore, we can characterize the computational complexity of the algorithm. 
Table 2: LeftCorner: identifies the left corner of $\Gamma^{\prime} \subseteq \Gamma$ (Thm. 5.5); the pseudocode of the dual function $\left(\delta_{0}^{\max }, \delta_{1}^{\min }\right)=$ Right Corner $\left(Q, c_{0}, c_{1}\right)$ is omitted for the sake of space.

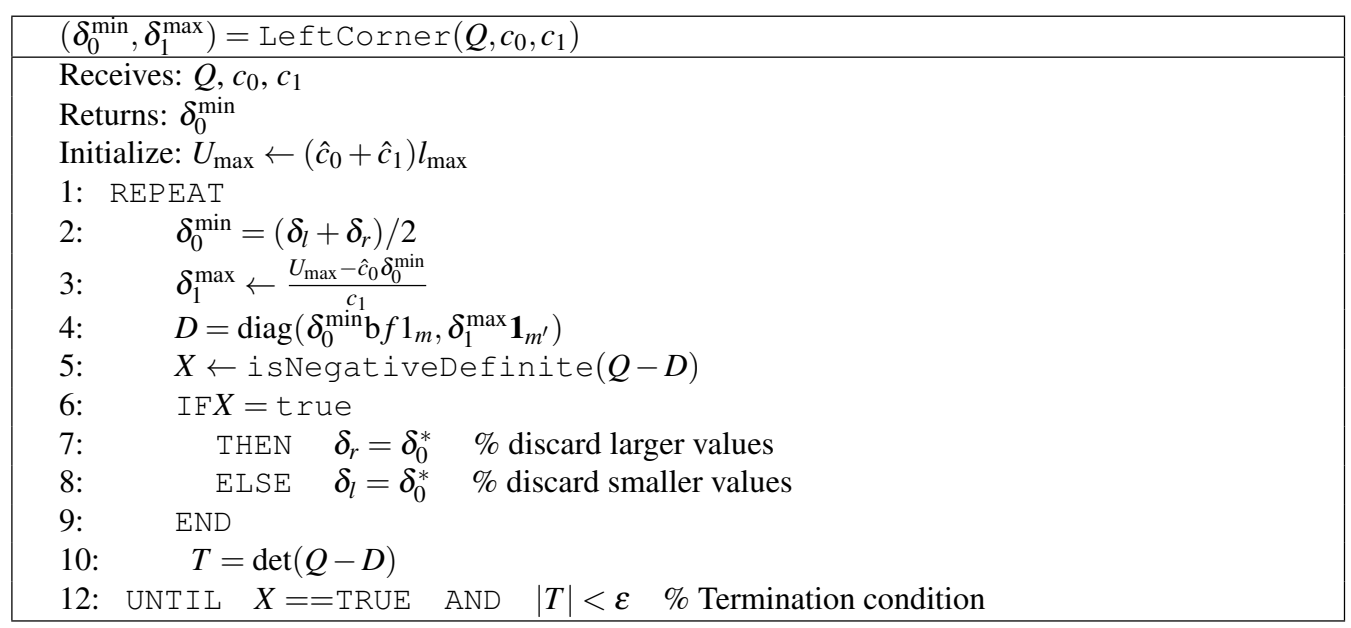

TheOREM 5.7 (Complexity) The time complexity of OptimalThreshold2D is $O\left(n^{1+\ell} \log _{2}(n / \varepsilon)\right)$ where $\ell=2.373$.

Proof. The number of iterations of the bisection search WHILE loop (lines 1 to 9 in Tab. 1) is $O\left(\log _{2}(n / \varepsilon)\right)$. This follows again from elementary properties of bisection search [32][Ch. 4, pp. 145]. In fact, the bisection search operates for $0 \leqslant U\left(\delta_{0}\right) \leqslant U_{l_{\max }}$ and $U_{l_{\max }}=l_{\max }\left(\hat{c}_{0}+\hat{c}_{1}\right)$. Finally, indeed, $l_{\max } \leqslant(n-1) \max _{i, j} q_{i j}$.

Same argument on the measure of the search intervals of BisectionThreshold, LeftCorner and RightCorner let us conclude that they require $O\left(\log _{2}(n / \varepsilon)\right)$ iterations of the REPEAT loop.

Finally, test isNegativeDefinite appearing in Threshold2D, LeftCorner and RightCorner requires the computation of $n-1$ determinants of the principal minors of $A-D$ at cost $O\left(n^{1+\ell}\right)$. Here $\ell$ is the exponent for fast matrix multiplication [55]. In the case of the Coppersmith-Winograd algorithm for fast matrix multiplication it holds $\ell=2.373$.

\subsection{Numerical Results}

In this section we present the results of numerical experiments in the case of interconnected stars networks, a sample network is depicted in Fig. 2b). In Figure 3a) we compare the ratio between the cost $U_{u}$ of the uniform curing rate vector, and the optimal cost $U^{*}=U\left(\Delta^{*}\right)$ obtained by solving the $2 D$ curing Prob. 5.3, by means of the OptimalThreshold2D. The uniform curing rate vector is $\Delta=\delta \mathbf{1}_{N}$, where $\delta$ is the value such that the threshold in (4.6) is attained. For this experiment, we consider that the infection spreads with rate $\beta_{V_{i}^{0} V_{j}^{0}}=\beta_{0}$ among the central communities and with rate $\beta_{V_{i} V_{i}^{0}}=\beta_{1}$ between a central node and a node in its adjacent terminal community, moreover we assume $c_{0}=c_{1}=1$. We consider that each terminal community has the same number of elements $k$. The computation is made for different values of $k$, for three different sample networks, with $m=50$ central nodes and $m^{\prime}=50$ terminal communities. Sample networks differ for the average degree of the central nodes. Central 
Table 3: BisectionThreshold: given feasible $\delta_{0}$, finds $\delta_{1}$ such that $\left(\delta_{0}, \delta_{1}\right)$ lies on the frontier of the feasibility region.

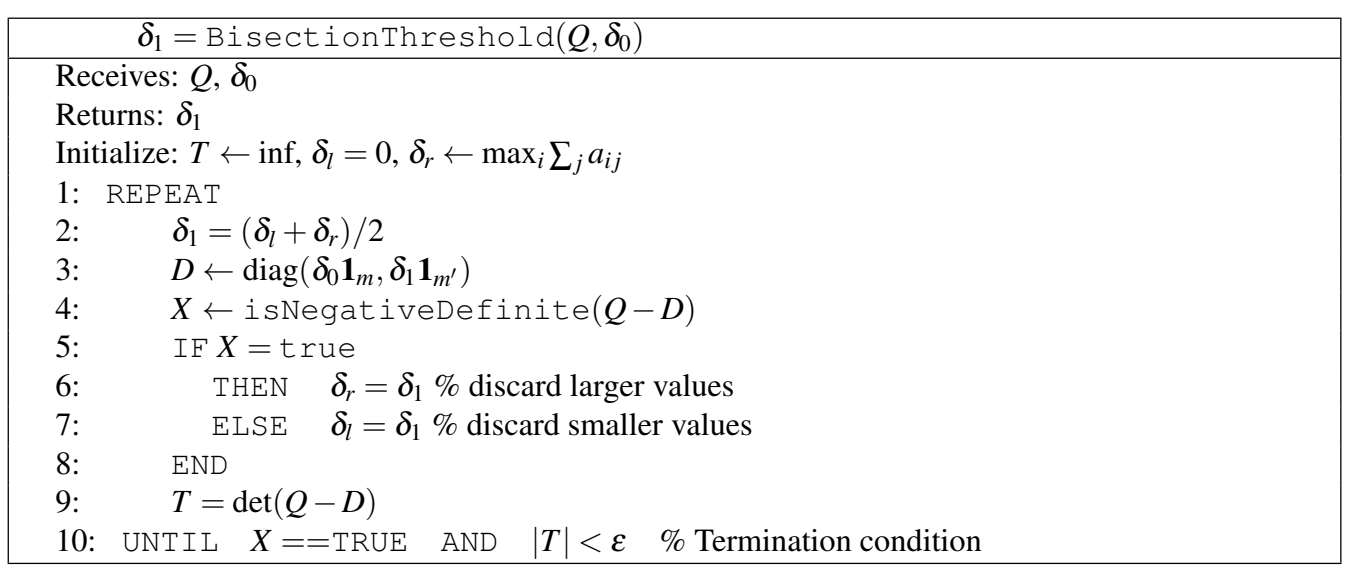

nodes are connected as Erdős-Rényi random graphs with $p=0.2, p=0.3, p=0.6$, respectively. The plot confirms that a larger gain is obtained, in terms of costs, by $2 D$ curing policy versus a uniform approach, in particular, the larger the denser the network, namely, for larger $p$ in our samples. For the interconnected stars networks samples, in particular, we observe one order of magnitude gain in the cost function. We see that the advantage increases as the number of elements $k$ increases, with a $\sqrt{k}$ shaped ratio (7.9) as derived in closed form for the case $m=m^{\prime}=2$. In Figure 3b) we have instead reported, only, on the optimal cost $U^{*}$ for different values of $c_{0}$ and $c_{1}$. In particular, we observe that the optimal cost appears to depend linearly on the community size $k$. Larger costs are incurred in the case when the coefficient $c_{0}$, related to the expenditure for the central nodes, is larger than $c_{1}$. This is in line with the fact that central communities are more connected than terminal communities, and consequently we need for more investments in such a way that the infection is kept subcritical.

In Tab. 4 we compare the performance of OptimalThreshold2D with an SDP solver, namely the SDPT 3 solver [35]. The SDPT3 solver generates a solution using a primal-dual interior-point algorithm which leverages on the infeasible path-following paradigm. As reported in Tab. 4, when the solver is applied to Prob. 5.3, we denote the corresponding solution as SDPT3 (2D). For the sake of comparison, we have reported also on the optimal solution derived with the same solver when curing rates are optimized per node (Prob 3.2), and we refer to this solution as SDPT3. The solution is provided on a graph with $m=m^{\prime}=50$ and $c_{0}=c_{1}=1$, for increasing values of the terminal community dimension $k$. We can observe that for the interconnected stars network, in the case of two infection rate levels, SDPT3, SDPT3 (2D) and OptimalThreshold2D provide similar values. This result suggests that, in this particular case, there is no advantage to treat each node with different curing policies: the general solution obtained using SDPT3 has similar performance as the one generated solving the $2 D$ formulation of the problem, i.e., by using OptimalThreshold2D or SDPT3 (2D) in the $2 D$ case. We observe also that the curing rate of terminal nodes appears insensitive to the increase of the terminal communities size $k$, as it can be seen, with a direct computation, in the examples 7.5 for the case with $m=1$ and $m=2$, respectively. In Tab. 5, same performance evaluation has been reported for the same sample graph and the same cost coefficients, but studying the case with more than two infection rate 

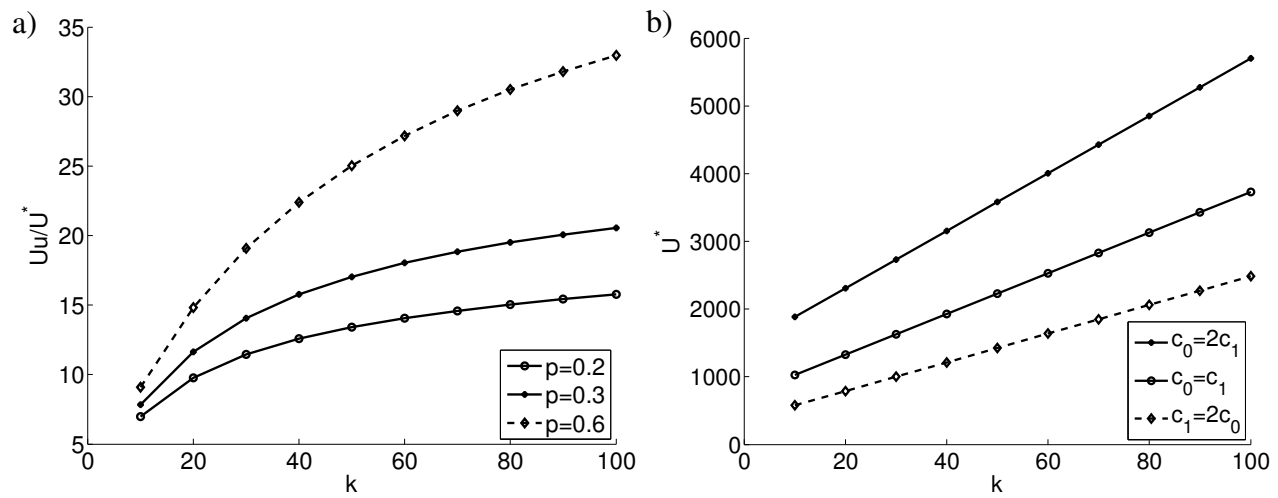

FIG. 3: (a) Ratio $U_{u} / U^{*}$ for increasing size $k$ of the terminal communities of interconnected star networks with $m=m^{\prime}=50$. The three curves refer to networks where the central nodes are connected as Erdôs-Rényi graphs generated for $p=0.2, p=0.3$ and $p=0.6$ respectively; $\beta_{0}=1, \beta_{1}=0.3, c_{0}=c_{1}=1$. (b) Cost function $U^{*}$ for increasing dimension $k$ of the terminal communities, $\beta_{0}=1, \beta_{1}=0.3$. The curves refer to the case $p=0.3$ in the cases $c_{0}=2 c_{1}, c_{0}=c_{1}$ and $2 c_{0}=c_{1}$, respectively.

levels; specifically a central node can eventually infect each of its adjacent central nodes with a different infection rate, also the infection rate between a central node and a terminal community can vary from a subgraph to another. The infection rates are generated as uniform random variables in the interval $(0.1,1.9)$ and $(0.03,0.57)$ for the speed of infection between central communities and between a central node and a terminal community respectively.

As seen there, by curing nodes with different curing policies it is possible to attain lower costs at larger values of $k$. This effect is depicted also in Fig. 4. In particular, again, the $2 D$ curing rates output of SDPT3 (2D) and OptimalThreshold2D show similar performance and the optimal curing rate of terminal communities appears insensitive to the increase of the terminal communities size. We observe that the relative advantage of the SDPT 3 tends to increase with the size of the terminal communities.

In the final set of experiments we study the case of a complete bipartite graph. We consider that the community whose curing rate is $\delta_{0}$, to which we refer to as the central community, has a fixed dimension $k_{0}=50$; instead, for the so-called terminal community, with $\delta_{1}$ curing rate, we consider increasing size $k_{1}=1,50,100,150,200$. In Fig. 5a) we report on the ratio between the cost obtained by using the uniform curing policy, namely $U_{u}$, and the optimal cost $U^{*}$ obtained by means of the OptimalThreshold2D, in the case of equal coefficients $c_{0}=c_{1}=1$. As expected, we can see that when $k_{1}=1$ we obtain an advantage in the use of the two-level curing strategy. Clearly, when the two communities have the same size there is no difference between the two costs; the ratio starts to grow again as the asymmetry in terms of communities dimensions, starts to increase again.

In Fig. 5b), we compare the effect of having two different cost coefficients, precisely $c_{1}=4 c_{0}$, with the case where they are the same, namely $c_{0}=c_{1}=1$, considering that their total amount is given and fixed a priori, that is $c_{0}+c_{1}=2$. A bias in the cost coefficient towards terminal community has a beneficial consequence because the optimal cost is lower than the case of equal coefficients, the advantage increases the larger the size of the terminal community. However, it is interesting to note that we would have obtained the same optimal cost if we interchanged the two coefficients, namely $c_{0}=4 c_{1}$ (see example 7.5). Basically, due to the specific topology of the network, we have an advantage in 
Table 4: Performance of OptimalThreshold2D, SDPT3 (2D) and SDPT3. The graph considered is an interconnected star network with $m=m^{\prime}=50$, where the connection between the central nodes are represented by a Erdős-Rényi graph generated with $p=0.2 ; c_{0}=c_{1}=1$ and the values of the weights are $\beta_{0}=1$, and $\beta_{1}=0.3$. In the SDPT 3 case, the values of $\delta_{i}^{*}, i=0,1$, represent the averaged value of the node-specific curing rates over a community.

\begin{tabular}{|c|ccc|ccc|ccc|}
\hline \hline & \multicolumn{2}{|c|}{ OptimalThreshold2D } & \multicolumn{3}{|c|}{ SDPT3 (2D) } & \multicolumn{3}{c|}{ SDPT3 } \\
\hline $\mathrm{k}$ & $U^{*}\left(10^{3}\right)$ & $\delta_{0}^{*}$ & $k \cdot \delta_{1}^{*}$ & $U^{*}\left(10^{3}\right)$ & $\delta_{0}^{*}$ & $k \cdot \delta_{1}^{*}$ & $U^{*}\left(10^{3}\right)$ & $\delta_{0}^{*}$ & $k \cdot \delta_{1}^{*}$ \\
\hline \hline 10 & 0.8057 & 13.116 & 0.29979 & 0.80572 & 13.1144 & 0.3 & 0.772 & 12.44 & 0.3 \\
\hline 20 & 1.1057 & 16.1163 & 0.29984 & 1.1057 & 16.1144 & 0.3 & 1.072 & 15.44 & 0.3 \\
\hline 30 & 1.4056 & 19.1222 & 0.29965 & 1.4057 & 19.1145 & 0.3 & 1.372 & 18.44 & 0.3 \\
\hline 40 & 1.7055 & 22.1294 & 0.29951 & 1.7057 & 22.1144 & 0.3 & 1.672 & 21.44 & 0.3 \\
\hline 50 & 2.0053 & 25.1354 & 0.29943 & 2.0057 & 25.1144 & 0.3 & 1.972 & 24.44 & 0.3 \\
\hline 60 & 2.3052 & 28.1414 & 0.29936 & 2.3057 & 28.1144 & 0.3 & 2.272 & 27.44 & 0.3 \\
\hline 70 & 2.6049 & 31.1578 & 0.29916 & 2.6057 & 31.1145 & 0.3 & 2.572 & 30.44 & 0.3 \\
\hline 80 & 2.9047 & 34.1792 & 0.29893 & 2.9057 & 34.1144 & 0.3 & 2.872 & 33.4401 & 0.3 \\
\hline 90 & 3.2043 & 37.1929 & 0.29882 & 3.2057 & 37.1144 & 0.3 & 3.172 & 36.4402 & 0.3 \\
\hline 100 & 3.5039 & 40.1319 & 0.29947 & 3.5057 & 40.1144 & 0.3 & 3.472 & 39.44 & 0.3 \\
\hline
\end{tabular}

Table 5: Performance of Optimal Threshold2D, SDPT3 (2D) and SDPT3, sample graphs are obtained from the same graph used in Tab. 4 and $c_{0}=c_{1}=1$. The infection rates are generated as uniform random variables in the interval $(0.1,1.9)$ and $(0.03,0.57)$, for rates between central communities and between a central node and a terminal community respectively. In the SDP T 3 case, the values of $\delta_{i}^{*}, i=0,1$, represent the averaged value of the node-specific curing rates over a community.

\begin{tabular}{|c|ccc|ccc|ccc|}
\hline \hline & \multicolumn{3}{|c|}{ Opt imalThreshold2D } & \multicolumn{3}{c|}{ SDPT3 (2D) } & \multicolumn{3}{c|}{ SDPT3 } \\
\hline $\mathrm{k}$ & $U^{*}\left(10^{3}\right)$ & $\delta_{0}^{*}$ & $k \cdot \delta_{1}^{*}$ & $U^{*}\left(10^{3}\right)$ & $\delta_{0}^{*}$ & $k \cdot \delta_{1}^{*}$ & $U^{*}\left(10^{3}\right)$ & $\delta_{0}^{*}$ & $k \cdot \delta_{1}^{*}$ \\
\hline \hline 10 & 1.9963 & 34.1309 & 0.57945 & 1.9963 & 34.1309 & 0.57945 & 1.9379 & 33.4102 & 0.53481 \\
\hline 20 & 2.5603 & 39.3993 & 0.5903 & 2.5603 & 39.3993 & 0.5903 & 2.4307 & 38.3384 & 0.51382 \\
\hline 30 & 3.1665 & 44.9001 & 0.61431 & 3.1666 & 44.9001 & 0.61431 & 2.9536 & 43.5669 & 0.51682 \\
\hline 40 & 3.7954 & 50.6431 & 0.63164 & 3.7957 & 50.6431 & 0.63164 & 3.4781 & 48.8125 & 0.51876 \\
\hline 50 & 4.4332 & 56.4137 & 0.64499 & 4.4336 & 56.4137 & 0.64499 & 4.0279 & 54.3098 & 0.52495 \\
\hline 60 & 5.1272 & 62.627 & 0.66528 & 5.1278 & 62.627 & 0.66528 & 4.6049 & 60.0804 & 0.53364 \\
\hline 70 & 5.8175 & 69.0209 & 0.67612 & 5.8184 & 69.0209 & 0.67612 & 5.1595 & 65.6263 & 0.53663 \\
\hline 80 & 6.4779 & 74.8633 & 0.68369 & 6.4792 & 74.8633 & 0.68369 & 5.6708 & 70.7388 & 0.53346 \\
\hline 90 & 7.1277 & 80.4688 & 0.68984 & 7.1298 & 80.4688 & 0.68984 & 6.1686 & 75.717 & 0.5295 \\
\hline 100 & 7.8361 & 87.1312 & 0.6959 & 7.839 & 87.1312 & 0.6959 & 6.7182 & 81.2132 & 0.53151 \\
\hline
\end{tabular}




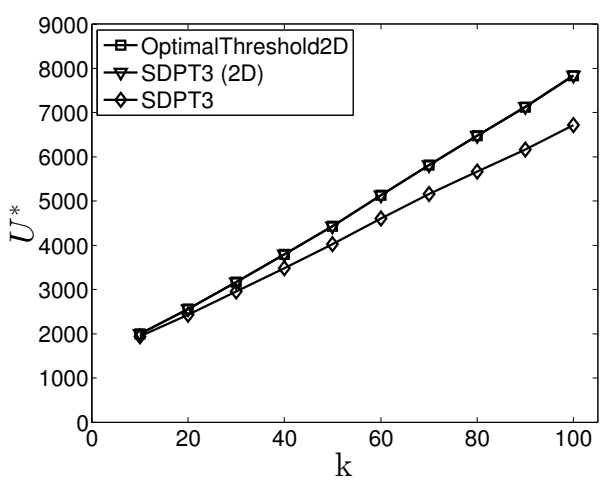

FIG. 4: Details of the costs in the case of uniform distribution of infection rates (see Tab. 5).

considering different cost coefficients for the two communities, instead of having them equal, if their total sum is fixed.

\section{Conclusions}

We have studied the problem of finding a non-uniform allocation of curing resources, within a networked population, at the minimum cost possible to avoid the epidemic from persisting indefinitely in the network. We have considered a mean-field approximation of an SIS model to study the diffusion of epidemics over a directed weighted graphs, capturing the possible asymmetric interactions, and the heterogeneity in the contagiousness. We have reported on the necessary and sufficient conditions for the extinction of the epidemics. These conditions are related to the sign of the stability modulus of a matrix encoding for the network structure and for the parameters of the model. Thus, such stability modulus represents the epidemic threshold of our system. Consequently, we have formulated a convex optimization problem for determining a cost-optimal curing policy, via a semidefinite programming approach, involving, the spectral properties of the network.

Then, we have specialized the theory to the case of equitable partitions, in order to model heterogeneous community networks that possess a certain degree of regularity in their connectivity; this choice has been motivated since communities are relevant non-trivial topological feature of complex networks, that often have a certain regularity in their structure. Thus, in this case, we were able to reduce the dimensionality of our optimization problem, that is useful since the size of many real-networks poses limitations in investigating their spectral properties.

At last, we have discussed on the special case of a two-dimensional curing policy, that can reflect, e.g., the case of different policy decisions for two different kinds of individual units (male and female, younger and elders, small villages and cities, firewall/gateways or clients in an enterprise network, etc.,...). With respect to this problem we have proposed an $\varepsilon$-approximation algorithm with polynomial complexity in the input size.

Fundings. This work has been partially supported by the European Commission within the framework of the CONGAS project FP7-ICT-2011-8-317672 (see http: / / www . congas-project . eu 

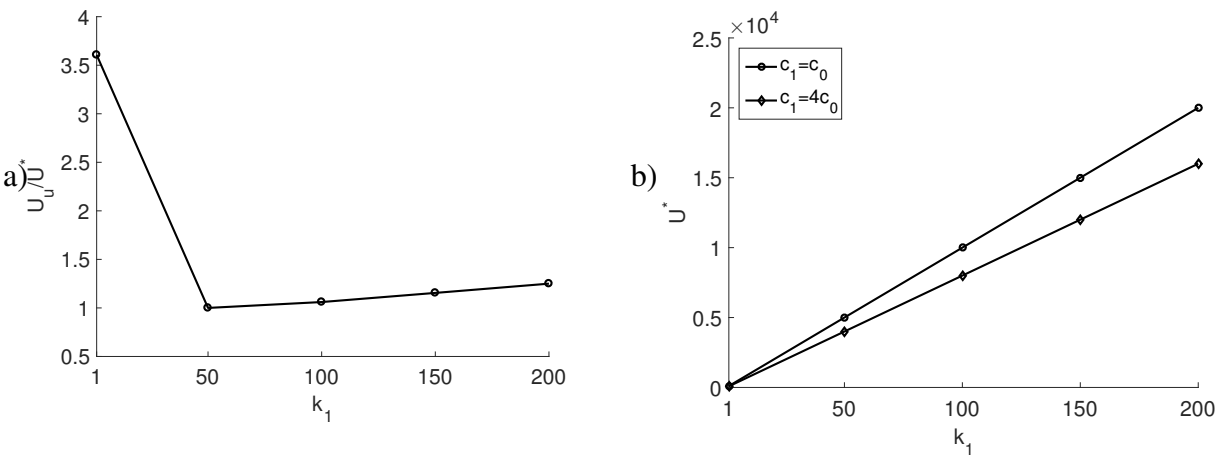

FIG. 5: (a) Ratio $U_{u} / U^{*}$ in the case of complete bipartite graphs for increasing size $k_{1}=1,50,100,150,200$ of the terminal community, and fixed size $k_{0}=50$ of the central community: $\beta=1, c_{0}=c_{1}=1$. (b) Cost function $U^{*}$ for increasing dimension $k_{1}$ of the terminal community and fixed size $k_{0}=50, \beta=1$. The two curves refer to the cases $c_{0}=c_{1}=1$ and $c_{1}=4 c_{0}$, respectively, in such a way that $c_{0}+c_{1}=2$.

\section{Appendix}

7.1 Dimensionality reduction of the dynamical system (2.2)

Let us define $q_{j m}^{T}$ as the element of $Q^{T}$ in position $(i, j)$. We know that

$$
q_{j m}^{T}=\frac{k_{j} c_{j m}^{\text {out }}}{\sqrt{k_{j} k_{m}}},
$$

hence

$$
c_{j m}^{\text {out }}=\frac{\sqrt{k_{j} k_{m}}}{k_{j}} q_{j m}^{T}=\left(\frac{k_{m}}{k_{j}}\right)^{1 / 2} q_{j m}^{T} .
$$

Thus we can write (4.5) in the following matrix form

$$
\frac{d \bar{P}(t)}{d t}=(\tilde{Q}-\bar{D}) \bar{P}(t)-\operatorname{diag}(\bar{P}(t)) \tilde{Q} \bar{P}(t),
$$

where $\widetilde{Q}=\operatorname{diag}\left(\frac{1}{\sqrt{k_{j}}}\right) Q^{T} \operatorname{diag}\left(\sqrt{k_{j}}\right)$. It is immediate that $\sigma\left(Q^{T}\right)=\sigma(\tilde{Q})$. 
By [20, Corollary 4.2], irrespective of the initial conditions of nodes in the same community, it is sufficient to compute the positive steady-state vector $\bar{P}_{\infty}$ of the reduced system (7.1) to obtain that of the original system (2.2). Indeed, as time elapses all nodes in the same community tend to have the same infection probabilities, thus the components of the steady-state vector $P_{\infty}$ corresponding to nodes in the same community are equal.

\subsection{Proof of Lemma 4.1}

Proof. i) We first prove that $A^{T} S^{T}=S^{T} Q^{T}$. In fact, if $i \in V_{h}$, it holds

$$
\begin{aligned}
\left(A^{T} S^{T}\right)_{i, j} & =\frac{c_{h j}^{\text {out }}}{\sqrt{k_{j}}}, \\
\left(S^{T} Q^{T}\right)_{i, j} & =\frac{1}{\sqrt{k_{h}}} q_{h j}^{T}=\frac{c_{h j}^{\text {out }}}{\sqrt{k_{j}}} .
\end{aligned}
$$

We further note that $\left(D S^{T}\right)_{i h}=\left(S^{T} \bar{D}\right)_{i h}=\frac{1}{\sqrt{k_{h}}} \delta_{h}$, if $i \in V_{h}$, otherwise $\left(D S^{T}\right)_{i h}=0$. Thus the statement holds.

ii) By using the result in $i$ ), the proof in [15, Thm. 2.2] applies.

\subsection{Proof of proposition 4.3}

We first need some technical facts to prove Prop. 4.3.

Proposition 7.1 Let $A$ be an $n \times n$ irreducible and non-negative matrix and let $D=\operatorname{diag}\left(\delta_{1}, \ldots, \delta_{n}\right)$. Then it holds:

i. $A-D$ is irreducible, for each $\left(\delta_{1}, \ldots, \delta_{n}\right)$.

ii. There exists an eigenvector $w$ of $A-D$ such that $w>0$ (i.e. each component $w_{i}>0, i=1, \ldots, n$ ) and the corresponding eigenvalue is $r(A-D)$, for each $\left(\delta_{1}, \ldots, \delta_{n}\right)$.

Proof. i. From [29]: a $n \times n$ matrix $A$ is said to be irreducible if for any proper subset $S \subseteq\{1, \ldots, n\}$ there exists $i \in S$ and $j \in S^{\prime}=\{1, \ldots, n\}-S$ such that $a_{i j} \neq 0$; since $A$ is irreducible, the definition applies immediately to $A-D$;

ii. See [29, Lemma 4.2].

With these background statements we prove Prop. 4.3. Proof. Basically, by Theorem 2.1, we have to show that

$$
r\left(A^{T}-D\right)=r(\tilde{Q}-\bar{D})=r\left(Q^{T}-\bar{D}\right)
$$

We first prove that

$$
r\left(Q^{T}-\bar{D}\right)=r\left(A^{T}-D\right) .
$$

Let $c \in \mathbb{R}$ such that both $a_{z z}^{T}-\delta_{z}+c \geqslant 0$, for all $z=1, \ldots, N$ and $q_{i i}^{T}-\bar{\delta}_{i}+c \geqslant 0$ for all $i=1 \ldots, n$. Let us define $A^{T}-D^{T}+c I_{N \times N}=\hat{A^{T}}$ and $Q^{T}-\bar{D}+c I_{n \times n}=\hat{Q^{T}} . \hat{A^{T}}$ and $\hat{Q^{T}}$ are non negative and irreducible matrices (see $i$ ) in Proposition 7.1). We order the eigenvalues of $\hat{Q}^{T}$ so that $\left|\lambda_{1}\left(\hat{Q}^{T}\right)\right| \geqslant$ $\left|\lambda_{2}\left(\hat{Q^{T}}\right)\right| \geqslant \ldots \geqslant\left|\lambda_{n}\left(\hat{Q^{T}}\right)\right|$, and similarly for $\hat{A^{T}}$. By the Perron-Frobenius theorem [56, Chapter 8], the eigenvalue of maximum modulus of an irreducible and non negative matrix is real and positive and its corresponding eigenvector, the Perron vector, is the unique (up to a factor) strictly positive eigenvector of the matrix. Hence there exists an eigenvector $w>0$ of $\hat{Q}^{T}$ corresponding to $\lambda_{1}\left(\hat{Q^{T}}\right)$, i.e. $w_{i}>0$, 
for all $i=1, \ldots, n$. Now, by $i i)$ in Lemma 4.1 and since $S^{T} I_{n \times n}=I_{N \times N} S^{T}$, we have that $S^{T} w>0$ is the eigenvector of $\hat{A^{T}}$ corresponding to $\lambda_{1}\left(\hat{Q^{T}}\right)$. However, because $S^{T} w$ is strictly positive, it must be the Perron vector of $\hat{A^{T}}$, consequently $\lambda_{1}\left(\hat{A^{T}}\right)=\lambda_{1}\left(\hat{Q^{T}}\right)$. Since

$$
r\left(\hat{Q^{T}}\right)=\lambda_{1}\left(\hat{Q^{T}}\right)=\lambda_{1}\left(\hat{A^{T}}\right)=r\left(\hat{A^{T}}\right),
$$

and

$$
r\left(Q^{T}-\bar{D}\right)+c=r\left(\hat{Q^{T}}\right)=r\left(\hat{A^{T}}\right)=r\left(A^{T}-D\right)+c,
$$

(7.5) is proved. Now we prove that

$$
r(\tilde{Q}-\bar{D})=r\left(Q^{T}-\bar{D}\right)
$$

Let the matrix $\Lambda=\operatorname{diag}\left(k_{i}\right), i=1, \ldots, n$. For any $n$-dimensional vector $v$ and scalar $\lambda \in \mathbb{C}$ we have that

$$
\begin{gathered}
(\tilde{Q}-\bar{D}) v=\lambda v \Leftrightarrow\left(\Lambda^{-\frac{1}{2}} Q \Lambda^{\frac{1}{2}}-\bar{D}\right) v=\lambda v \Leftrightarrow \\
\left(Q \Lambda^{\frac{1}{2}}-\bar{D} \Lambda^{\frac{1}{2}}\right) v=\lambda \Lambda^{\frac{1}{2}} v \Leftrightarrow \\
(Q-\bar{D})\left(\Lambda^{\frac{1}{2}} v\right)=\lambda\left(\Lambda^{\frac{1}{2}} v\right),
\end{gathered}
$$

hence $\lambda \in \sigma(\tilde{Q}-\bar{D}) \Longleftrightarrow \lambda \in \sigma(Q-\bar{D})$, so that (7.7) is verified. In conclusion, from (7.7) and (7.5) it follows (7.4).

\subsection{Proof of proposition 5.4}

Proof. i. Let $\delta_{i}=0$ for some $i=1, \ldots n$ and assume that $\lambda_{1}(A-D)<0$ : for the vector $\mathbf{e}_{i}$ of the canonical basis it holds $\mathbf{e}_{i}^{T}(A-D) \mathbf{e}_{i}=\mathbf{e}_{i}^{T} A \mathbf{e}_{i} \geqslant 0$ which is a contradiction.

ii. The eigenvalues of such kind of matrix vary with continuity with the entries of the matrix [56, Appendix D].

iii. Let us consider $c>0$ such that $-d_{i i}+c \geqslant 0$ for all $i=1, \ldots, n$. Then, $A-D+c I$ is non-negative irreducible and $\lambda_{1}\left(A-D+c \cdot I_{n}\right)$ is actually its Perron-Frobenius eigenvalue [56, Chapter 8]. Now, we can write for any $\varepsilon>0$

$$
\lambda_{1}\left(A-D+\varepsilon \operatorname{diag}\left(\mathbf{e}_{i}\right)\right)=\lambda_{1}\left(A-D+\varepsilon \operatorname{diag}\left(\mathbf{e}_{i}\right)+c I_{n}\right)-c>\lambda_{1}\left(A-D+c I_{n}\right)-c=\lambda_{1}(A-D),
$$

where the strict majorization holds because the Perron-Frobenius eigenvalue is strictly increasing in any entry of the matrix $[57,56]$.

\subsection{Examples}

1. A simple example of the optimal solution for the case of a community network is that of a star graph, where we have two communities, one formed by the central node and the other by the leaf nodes. Assuming that the infection rate is $\beta$, we have to find the value of $\delta_{0}$ and $\delta_{1}$ for which $\beta Q-D$ has the maximal eigenvalue which is equal to zero. The characteristic polynomial of $\beta Q-D$ for a star graph with $k$ leaves is

$$
p_{\lambda}(\beta Q-D)=\lambda^{2}+\left(\delta_{0}+\delta_{1}\right) \lambda+\delta_{0} \delta_{1}-\beta^{2} k .
$$


We observe that $\lambda=0$ belongs to the spectrum of $\beta Q-D$ if and only if $\delta_{0}=\beta^{2} k / \delta_{1}$. This also ensures that the second eigenvalue is negative and, consequently, $\lambda=0$ must be the largest eigenvalue of $\beta Q-D$. Thus replacing the value of $\delta_{0}$ obtained above in

$$
U\left(\delta_{0}, \delta_{1}\right)=c_{0} \delta_{0}+c_{1} k \delta_{1},
$$

and setting to zero the following derivative

$$
U^{\prime}\left(\delta_{1}\right)=-\frac{c_{0} \beta^{2} k^{3}}{\delta_{1}^{2}}+c_{1} k
$$

we have that the linear cost optimization is solved for

$$
\delta_{0}=\beta k \sqrt{\frac{c_{1}}{c_{0}}}, \quad \delta_{1}=\beta \sqrt{\frac{c_{0}}{c_{1}}},
$$

which in turn provides the optimal cost

$$
U^{*}=\beta k\left(c_{0} \sqrt{\frac{c_{1}}{c_{0}}}+c_{1} \sqrt{\frac{c_{0}}{c_{1}}}\right)=2 \beta k\left(\sqrt{c_{1} c_{0}}\right) .
$$

We observe that the optimal cost is linear in the terminal community size $k$.

In the case of a uniform curing policy, where all nodes are cured at rate $\delta$, we have that the value of $\delta$ such that $\lambda=0$ is the largest eigenvalue of $\beta Q-D$ is equal to $\beta \sqrt{k}$, thus the value $U_{u}$ of the total cost is

$$
U_{u}=\beta \sqrt{k}\left(c_{0}+c_{1} k\right) .
$$

It is easy to see that the ratio $U_{u} / U^{*}$ is increasing in $(1, \infty)$, moreover we can observe that

$$
\frac{U_{u}}{U^{*}}=O(\sqrt{k}) .
$$

Hence it is clear that we have an advantage in considering a two-level curing policy, with respect to the uniform curing policy.

2. Now we consider an interconnected star network with two linked central nodes, where each terminal community has the same number of elements $k$. We set $\beta$ as the infection rate between the central nodes and $\varepsilon \beta$ the infection rate between a central node and a node in its adjacent terminal community, where $\varepsilon>0$. After computing the characteristic polynomial of $\beta Q-D$ we can see that the zero eigenvalue belongs to the spectrum of $\beta Q-D$ provided that

$$
\delta_{0}^{2} \delta_{1}^{2}-2 \beta^{2} \delta_{0} \delta_{1} \varepsilon^{2} k+\varepsilon^{4} \beta^{4} k^{2}-\beta^{2} \delta_{1}^{2}=0 .
$$

The values of $\delta_{0}$ for which $\lambda=0$ corresponds to the largest eigenvalue of $\beta Q-D$ is equal to $\delta_{0}=$ $\frac{\beta^{2} \varepsilon^{2} k}{\delta_{1}}+\beta$ and the linear cost optimization is solved for

$$
\delta_{0}=\beta\left(\varepsilon k \sqrt{\frac{c_{1}}{c_{0}}}+1\right), \quad \delta_{1}=\varepsilon \beta \sqrt{\frac{c_{0}}{c_{1}}} .
$$

Consequently the optimal cost is

$$
U^{*}=2 \beta c_{0}\left(\varepsilon k \sqrt{\frac{c_{1}}{c_{0}}}+1\right)+2 c_{1} k \sqrt{\frac{c_{0}}{c_{1}}} \varepsilon \beta=2 \beta \sqrt{c_{0} c_{1}}\left(\varepsilon(k+1)+c_{0}\right) .
$$


In the case of a uniform curing policy we have that the value of $\delta$ such that $\lambda=0$ is the largest eigenvalue is $\left(\beta+\sqrt{\beta^{2}+4 \beta^{2} \varepsilon^{2} k}\right) / 2$ and the value of the total cost is

$$
U_{u}=c_{0}\left(\beta+\sqrt{\beta^{2}+4 \beta^{2} \varepsilon^{2} k}\right)+c_{1} k\left(\beta+\sqrt{\beta^{2}+4 \beta^{2} \varepsilon^{2} k}\right) .
$$

The ratio $U_{u} / U^{*}$ is increasing in $(0, \infty)$, and again we have that

$$
\frac{U_{u}}{U^{*}}=O(\sqrt{k}) .
$$

3. Now we consider a complete bipartite graph. Basically, we have two communities and we denote by $k_{0}$ the number of elements in the community whose nodes have recovery rate $\delta_{0}$ and $k_{1}$ the number of elements in the community whose nodes has recovery rate $\delta_{1}$. The optimal curing rates are

$$
\delta_{0}=\beta k_{1} \sqrt{\frac{c_{1}}{c_{0}}}, \quad \delta_{1}=\beta k_{0} \sqrt{\frac{c_{0}}{c_{1}}},
$$

and the optimal cost is

$$
U^{*}=c_{0} k_{0} \beta k_{1} \sqrt{\frac{c_{1}}{c_{0}}}+c_{1} k_{1} \beta k_{0} \sqrt{\frac{c_{0}}{c_{1}}} .
$$

In the case of a uniform curing policy the value of $\delta$ such that $\lambda=0$ is the largest eigenvalue is

$$
\delta=\beta \sqrt{k_{0} k_{1}},
$$

and the cost is

$$
U_{u}=c_{0} k_{0} \beta \sqrt{k_{0} k_{1}}+c_{1} k_{1} \beta \sqrt{k_{0} k_{1}} .
$$

In this case the asymptotic behavior of $U_{U} / U^{*}$ for high values of $k_{0}$ and $k_{1}$ depends on the direction in which we move, thus we can not say anything in this regard.

\section{References}

[1] Romualdo Pastor-Satorras, Claudio Castellano, Piet Van Mieghem, and Alessandro Vespignani. Epidemic processes in complex networks. Reviews of modern physics, 87(3):925, 2015.

[2] Yan Wan, Sandip Roy, and Ali Saberi. Designing spatially heterogeneous strategies for control of virus spread. Systems Biology, IET, 2(4):184-201, 2008.

[3] B Aditya Prakash, Lada Adamic, TJ Iwashyna, Hanghang Tong, and Christos Faloutsos. Fractional immunization in networks. Austin, Texas, USA, 2013.

[4] Christian Borgs, Jennifer Chayes, Ayalvadi Ganesh, and Amin Saberi. How to distribute antidote to control epidemics. Random Structures \& Algorithms, 37(2):204-222, 2010.

[5] Eric Gourdin, Jasmina Omic, and Piet Van Mieghem. Optimization of network protection against virus spread. In Design of Reliable Communication Networks (DRCN), 2011 8th International Workshop on the, pages 86-93. IEEE, 2011.

[6] Faryad Darabi Sahneh and Caterina M Scoglio. Optimal information dissemination in epidemic networks. In Decision and Control (CDC), 2012 IEEE 51st Annual Conference on, pages 16571662. IEEE, 2012. 
[7] P. Van Mieghem, J. Omic, and R. Kooij. Virus spread in networks. Networking, IEEE/ACM Tran. on, 17(1):1-14, Feb 2009.

[8] V. M. Preciado, M. Zargham, C. Enyioha, A. Jadbabaie, and G. Pappas. Optimal vaccine allocation to control epidemic outbreaks in arbitrary networks. CoRR, abs/1303.3984, 2013.

[9] Victor M Preciado, Michael Zargham, Chinwendu Enyioha, Ali Jadbabaie, and George J Pappas. Optimal resource allocation for network protection against spreading processes. Control of Network Systems, IEEE Transactions on, 1(1):99-108, 2014.

[10] Chinwendu Enyioha, Ali Jadbabaie, Victor Preciado, and George J Pappas. Distributed resource allocation for epidemic control. arXiv preprint arXiv:1501.01701, 2015.

[11] Kimon Drakopoulos, Asuman Ozdaglar, and John N Tsitsiklis. When is a network epidemic hard to eliminate? arXiv preprint arXiv:1510.06054, 2015.

[12] Kimon Drakopoulos, Asuman Ozdaglar, and John N Tsitsiklis. A lower bound on the performance of dynamic curing policies for epidemics on graphs. arXiv preprint arXiv:1510.06055, 2015.

[13] Stefano Boccaletti, Vito Latora, Yamir Moreno, Martin Chavez, and D-U Hwang. Complex networks: Structure and dynamics. Physics reports, 424(4):175-308, 2006.

[14] Allen J Schwenk. Computing the characteristic polynomial of a graph. In Graphs and Combinatorics, pages 153-172. Springer, 1974.

[15] C. Godsil. Feasibility conditions for the existence of walk-regular graphs. Linear Algebra and its Applications, 30:15-61, 1980.

[16] Delio Mugnolo. Semigroup methods for evolution equations on networks. Springer, 2014.

[17] Duncan J Watts, Roby Muhamad, Daniel C Medina, and Peter S Dodds. Multiscale, resurgent epidemics in a hierarchical metapopulation model. Proceedings of the National Academy of Sciences of the United States of America, 102(32):11157-11162, 2005.

[18] Robert M May and Roy M Anderson. Spatial heterogeneity and the design of immunization programs. Mathematical Biosciences, 72(1):83-111, 1984.

[19] Steven Riley, Christophe Fraser, Christl A Donnelly, Azra C Ghani, Laith J Abu-Raddad, Anthony J Hedley, Gabriel M Leung, Lai-Ming Ho, Tai-Hing Lam, Thuan Q Thach, et al. Transmission dynamics of the etiological agent of sars in hong kong: impact of public health interventions. Science, 300(5627):1961-1966, 2003.

[20] Stefano Bonaccorsi, Stefania Ottaviano, Delio Mugnolo, and Francesco De Pellegrini. Epidemic outbreaks in networks with equitable or almost-equitable partitions. SIAM Journal of Applied Mathematics, 75(6):2421 - 2443, 2015.

[21] Faryad Darabi Sahneh, Caterina Scoglio, and Piet Van Mieghem. Generalized epidemic meanfield model for spreading processes over multilayer complex networks. Networking, IEEE/ACM Transactions on, 21(5):1609-1620, 2013.

[22] Piet Van Mieghem. Decay towards the overall-healthy state in sis epidemics on networks. arXiv preprint arXiv:1310.3980, 2013. 
[23] Moez Draief and Laurent Massouli. Epidemics and rumours in complex networks. Cambridge University Press, 2010.

[24] P Van Mieghem. Approximate formula and bounds for the time-varying susceptible-infectedsusceptible prevalence in networks. Physical Review E, 93(5):052312, 2016.

[25] Piet Van Mieghem. Exact markovian sir and sis epidemics on networks and an upper bound for the epidemic threshold. arXiv preprint arXiv:1402.1731, 2014.

[26] Piet Van Mieghem. Exact markovian sir and sis epidemics on networks and an upper bound for the epidemic threshold. arXiv preprint arXiv:1402.1731, 2014.

[27] E. Cator and P. Van Mieghem. Nodal infection in Markovian SIS and SIR epidemics on networks are non-negatively correlated. Physical Review E, 89(5):052802, 2014.

[28] Piet Van Mieghem and Jasmina Omic. In-homogeneous virus spread in networks. arxiv: 1306.2588, 2013.

[29] Ana Lajmanovich and James A. Yorke. A deterministic model for Gonorrhea in a nonhomogeneous population. Mathematical Biosciences, 28(34):221 - 236, 1976.

[30] Nathaniel Hupert, Jason Cuomo, Mark A Callahan, Alvin I Mushlin, and Stephen S Morse. Community-based mass prophylaxis: A planning guide for public health preparedness. US Department of Health and Human Services, Agency for Healthcare Research and Quality, 2004.

[31] S. P. Boyd. Semidefinite programming. SIAM review, 38:49-95, 1994.

[32] S.P. Boyd and L. Vandenberghe. Convex optimization. Cambridge University Press, 2004.

[33] Abraham Berman and Robert J Plemmons. Nonnegative matrices in the mathematical sciences. SIAM, 1994.

[34] Fuzhen Zhang. Matrix theory: basic results and techniques. Springer Science \& Business Media, 2011.

[35] R. H. Tütüncü, K. C. Toh, and M. J. Todd. Solving semidefinite-quadratic-linear programs using SDPT3. Mathematical Programming, 95(2):189-217, 2003.

[36] Frank Ball, Tom Britton, Thomas House, Valerie Isham, Denis Mollison, Lorenzo Pellis, and Gianpaolo Scalia Tomba. Seven challenges for metapopulation models of epidemics, including households models. Epidemics, 10:63-67, 2015.

[37] Ilkka Hanski and Otso Ovaskainen. Metapopulation theory for fragmented landscapes. Theoretical population biology, 64(1):119-127, 2003.

[38] Naoki Masuda. Effects of diffusion rates on epidemic spreads in metapopulation networks. New Journal of Physics, 12(9):093009, 2010.

[39] Frank Ball, Denis Mollison, and Gianpaolo Scalia-Tomba. Epidemics with two levels of mixing. The Annals of Applied Probability, pages 46-89, 1997. 
[40] Joshua V Ross, Thomas House, and Matt J Keeling. Calculation of disease dynamics in a population of households. PLoS One, 5(3):e9666, 2010.

[41] Frank Ball and Peter Neal. A general model for stochastic sir epidemics with two levels of mixing. Mathematical biosciences, 180(1):73-102, 2002.

[42] Lorenzo Pellis, Frank Ball, and Pieter Trapman. Reproduction numbers for epidemic models with households and other social structures. i. definition and calculation of $\mathrm{r} 0$. Mathematical biosciences, 235(1):85-97, 2012.

[43] Frank Ball and Peter Neal. Network epidemic models with two levels of mixing. Mathematical biosciences, 212(1):69-87, 2008.

[44] Ball Frank, Sirl David, Trapman Pieter, et al. Threshold behaviour and final outcome of an epidemic on a random network with household structure. Advances in Applied Probability, 41(3):765-796, 2009.

[45] Huijuan Wang, Qian Li, Gregorio D?Agostino, Shlomo Havlin, H Eugene Stanley, and Piet Van Mieghem. Effect of the interconnected network structure on the epidemic threshold. Physical Review E, 88(2):022801, 2013.

[46] Stefano Bonaccorsi, Stefania Ottaviano, Francesco De Pellegrini, Annalisa Socievole, and Piet Van Mieghem. Epidemic outbreaks in two-scale community networks. Phys. Rev. E, 90:012810, Jul 2014.

[47] Michelle Girvan and Mark EJ Newman. Community structure in social and biological networks. Proceedings of the national academy of sciences, 99(12):7821-7826, 2002.

[48] Ian Stewart, Martin Golubitsky, and Marcus Pivato. Symmetry groupoids and patterns of synchrony in coupled cell networks. SIAM Journal on Applied Dynamical Systems, 2(4):609-646, 2003 .

[49] Martin Golubitsky, Ian Stewart, and Andrei Török. Patterns of synchrony in coupled cell networks with multiple arrows. SIAM Journal on Applied Dynamical Systems, 4(1):78-100, 2005.

[50] Amirreza Rahmani, Meng Ji, Mehran Mesbahi, and Magnus Egerstedt. Controllability of multiagent systems from a graph-theoretic perspective. SIAM Journal on Control and Optimization, 48(1):162-186, 2009.

[51] Cesar O Aguilar and Bahman Gharesifard. On almost equitable partitions and network controllability. In American Control Conference (ACC), 2016, pages 179-184. IEEE, 2016.

[52] Arkadi Nemirovski. Advances in convex optimization: Conic programming. In In Proceedings of International Congress of Mathematicians, pages 413-444, 2007.

[53] Albert-László Barabási and Réka Albert. Emergence of scaling in random networks. science, 286(5439):509-512, 1999.

[54] Cong Li, Ruud van de Bovenkamp, and Piet Van Mieghem. Susceptible-infected-susceptible model: A comparison of n-intertwined and heterogeneous mean-field approximations. Physical Review E, 86(2):026116, 2012. 